\title{
Reconstrução da estrutura de volatilidade de uma carteira de ativos de renda variável
}

\author{
Mariela Fernández
}

\author{
Dissertação apresentada \\ ao \\ Instituto de Matemática e Estatística \\ da \\ Universidade de São Paulo \\ para \\ obtenção do grau \\ de \\ Mestre em Matemática
}

Área de Concentração: Matemática Aplicada Orientador: Prof. Dr. Pedro Paulo Schirmer

Durante a elaboração deste trabalho a autora recebeu apoio financeiro da CAPES

São Paulo - julho - 2003 


\section{Reconstrução da estrutura de volatilidade de uma carteira de ativos de renda variável}

Este exemplar corresponde à redação final da dissertação devidamente corrigida e defendida por Mariela Fernández e aprovada pela comissão julgadora.

São Paulo, agosto de 2003.

Banca examinadora:

- Prof. Dr. Pedro Paulo Schirmer (Orientador) - IME-USP

- Prof. Dr. Henrique Von Dreifus - IME-USP

- Prof. Dr. Gerson Francisco - IFT-UNESP 
A mi hermano por confiar en mi desde un comienzo.

A mi mamá y a mi abuela por ser mis ejemplos de vida. 


\section{Agradecimentos}

O mais difícil de escrever os agradecimentos é fazê-lo de maneira sinóptica. São muitas as pessoas com as quais me sinto agradecida: família, amigos ( os daqui e os de longe), professores, colegas, ... Mas, para ser breve, mencionarei os que estiveram envolvidos diretamente no desenvolvimento deste trabalho.

Agradeço à minha família e a Silvina, a "irmã" que ganhei no Brasil, pela infinita ajuda dada.

Agradeço ao meu orientador, Pedro Paulo, por confiar em mim e incentivar-me a continuar, assim como por todos os conhecimentos brindados.

Agradeço a meus colegas de mestrado Marcelo e Márcio pelas horas de estudo compartilhadas.

Agradeço a Felipe por ser tão fã do $\mathrm{AT}_{\mathrm{E} X} \mathrm{X}$ quanto eu e sempre ter boas dicas para compartilhar.

Agradeço a Jorge por sua paciência para responder minhas dúvidas de finanças.

Agradeço à CAPES pelo suporte financeiro. 


\section{Resumo}

O objetivo principal da presente dissertação é reconstruir a volatilidade de uma carteira de ativos cujas estruturas de volatilidade são conhecidas. Especificamente, tal questão consiste em sintetizar as volatilidades de um número finito de ativos num único índice financeiro que os integre, possibilitando o cálculo de opções sobre tal índice.

A dissertação se divide em duas partes, uma teórica e uma outra experimental. Na fase teórica foram estudados temas de cálculo estocástico (processos de Itô), otimização e análise assintótica (método de Laplace). $\mathrm{Na}$ fase experimental, foi aplicado o modelo obtido para o cálculo da volatilidade implícita de uma carteira de ações do mercado brasileiro. 


\section{Abstract}

The main purpose of this work is reconstructing the volatility of a basket of stocks whose structures are known. Specifically, we are synthezing the volatilities of a finite number of stocks in a single financial index which integrates them, enabling to price index options.

This work is divided in two stages, a theoretical one and an experimental one. In the first stage, topics of Stochastic Calculus (Itô process), Optimization and Asymptotic Analysis (Laplace's method) were studied. In the second one, the defined model was applied for computing the implied volatility of a basket of Brazilian shares. 


\section{Sumário}

Introdução $\quad$ i

1 Modelos de volatilidade 1

1.1 Estruturas de volatilidade . . . . . . . . . . . . . . . . 1

1.2 Modelo matemático da carteira . . . . . . . . . . . . 2

2 Reconstrução da estrutura de volatilidade do índice $\quad 5$

2.1 Volatilidade local . . . . . . . . . . . . . . . 5

2.2 Aproximação assintótica . . . . . . . . . . . . . . . . 10

2.3 A configuração mais provável de atingir o índice . . . . . . 14

3 Volatilidade implícita 20

3.1 Fórmula de Dupire . . . . . . . . . . . . . . . 20

3.2 Relação assintótica entre volatilidade local e implícita . . . . 24

3.3 Volatilidade implícita da carteira . . . . . . . . . . . . 29

4 Aplicação para o mercado brasileiro de derivativos 31

4.1 Estimação da correlação entre os ativos . . . . . . . . . . . 31

4.1 .1 Estimativa intra-diária . . . . . . . . . . . . . 31

4.1 .2 Estimativa diária . . . . . . . . . . . . . . . 32

4.2 Smiles de volatilidade . . . . . . . . . . . . 34

$\begin{array}{ll}\text { Conclusão } & 40\end{array}$ 
A Apêndice 41

A.1 Método de Laplace . . . . . . . . . . . . . . . . . . 41

A.2 Algoritmos . . . . . . . . . . . . . . . . 47

Referências Bibliográficas $\quad 49$ 


\section{Introdução}

"We have not succeeded in answering all our problems. The answers we have found only serve to raise a whole set of new questions. In some ways we feel we are as confused as ever, but we believe we are confused on a higher level and about more important things".

A volatilidade é a medida de nossa incerteza quanto aos retornos proporcionados por ativos de renda variável. Sendo um parâmetro essencial na descrição de um ativo líquido, a sua determinação é fundamental. Como não pode ser observada diretamente do mercado, já têm sido desenvolvidas diversas ferramentas matemática para estimá-la.

No caso da modelagem de ativos líquidos, é muito utilizada a volatilidade implícita obtida diretamente dos dados do mercado. Mas no caso de uma carteira, a obtenção de tal volatilidade não sempre é tão simples, pois a carteira não é um ativo com valor no mercado. Além disto, seu cálculo envolve um estudo integral dos retornos dos ativos que a comporem, considerando as correlações entre tais ativos.

Tanto a complexidade de dito cálculo quanto a importância do conhecimento da volatilidade fundamentam este trabalho. O objetivo principal é compreender e conseguir implementar computacionalmente os resultados apresentados em [Ave02], procurando obter a fórmula para a volatilidade implícita de uma carteira de ações. Especificamente, pretendemos sintetizar as volatilidades smile das ações que comporem a carteira numa volatilidade 
smile da carteira. No seguinte esquema damos uma idéia gráfica do nosso objetivo principal, que está presente, como motivação, na teoria apresentada nos capítulos 1,2 e 3 .

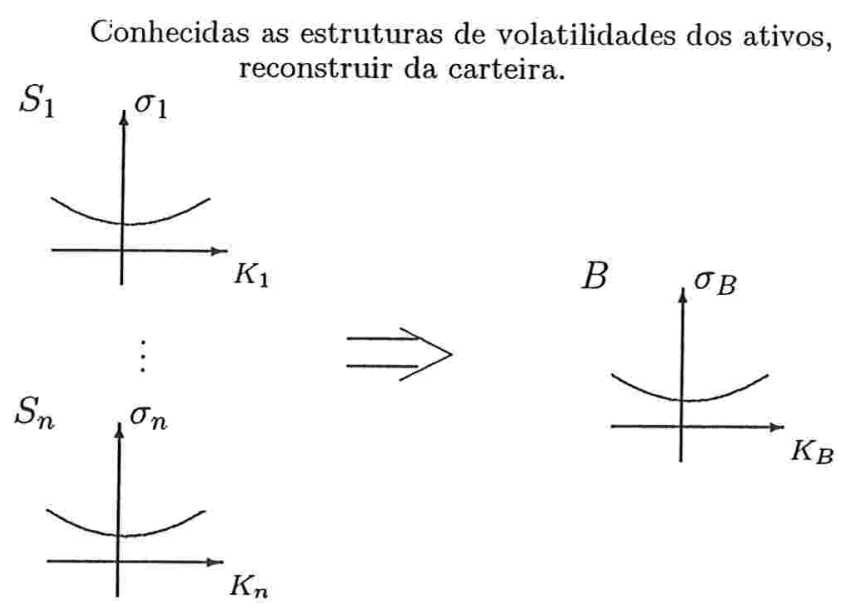

Sendo $B$ a carteira de ações $S_{i}, K_{i}$ os valores de exercício e $\sigma$ as respectivas volatilidades.

Fazemos ênfase em que uma fase do trabalho é calcular a volatilidade local da carteira a partir da estocástica, sendo esta fase relevante por sua complexidade matemática, pois envolve questões de análise assintótica e otimização. Também tem um grau de complexidade importante sua interpretação financeira, entendendo o como conectar um único índice com uma única configuração ótima de preços dos ativos. Por outro lado, como a motivação é obter fórmulas aplicáveis no mercado, temos uma outra fase, não menos importante, que relaciona a volatilidade local com a implícita por meio de um resultado matemático muito interessante.

O roteiro do trabalho será o seguinte. Começamos apresentando, em um contexto matemático, os conceitos essenciais para a presente dissertação. No segundo capítulo damos a definição matemática da volatilidade local da carteira e determinamos expressões equivalentes para facilitar o seu estudo. Logo, aplicamos ferramentas de análise assintótico e resolvemos um 
problema de minimização para ter uma aproximação da volatilidade local calculável numericamente. No terceiro capítulo apresentamos a Fórmula de Dupire devido a sua importância teórica, já que prova que faz sentido pensar em uma única volatilidade local que modela dinamicamente o processo de preços de um ativo; continuamos com o estudo de um resultado que permite relacionar a volatilidade local e a implícita, para logo terminar deduzindo as fórmulas que nos permitem fazer cálculos com dados reais. Finalmente, no quarto capítulo, aplicamos os resultados obtidos a dados do mercado brasileiro. 


\section{Capítulo 1}

\section{Modelos de volatilidade}

Como este trabalho se centra nos conceitos de volatilidade local, implícita e estocástica e suas relações, no presente capítulo revisamos suas noções. Também definimos o modelo matemático que empregamos para estudar a volatilidade da carteira.

\subsection{Estruturas de volatilidade}

Existem diferentes tipos de volatilidades segundo o modelo utilizado para calculá-a:

- "volatilidade histórica", é calculada a partir dos dados das variações que tiveram os preços no passado;

- "volatilidade implícita", comumente chamada smile, é obtida da fórmula de Black e Scholes para uma opção;

- "volatilidade estocástica", é o coeficiente de difusão do processo de preços;

- "volatilidade local", é o valor esperado da volatilidade estocástica condicionado por um estado do subjacente. 
Embora neste trabalho definimos matematicamente tais volatilidades mais adiante, é interessante salientar que elas se diferenciam pela sua característica como função. Mais especificamente, a volatilidade histórica é um número fixo para a evolução toda dos preços. A volatilidade implícita é calculada em função do tempo de vencimento de uma opção e de seu valor de exercício, utilizando o respectivo valor de opção fornecido pelo mercado; podemos interpretá-la como uma função discreta, já que o mercado, na realidade, só aprecifica para alguns valores de exercício e algumas maturidades. Por outra parte, a volatilidade estocástica é um processo que define, para cada instante de tempo, uma variável aleatória dos retornos do ativo. Por último, a volatilidade local é uma função determinística do tempo e do preço. Observamos que obtemos melhores modelos da dinâmica dos preços com o custo de perder simplicidade na função que representa a estrutura da volatilidade.

\subsection{Modelo matemático da carteira}

Nesta seção definimos, em um contexto matemático, uma carteira de ativos de renda variável, apresentando os conceitos e a notação que será utilizada no trabalho todo.

Dada uma carteira de $n$ ativos, ativos líquidos como por exemplo ações, com processo de preços $S_{i}(t)$ na qual a quantidade de cada ativo está determinada por um valor $w_{i}$, o índice da carteira é

$$
B(t, \mathbf{S}):=\sum_{i=1}^{n} w_{i} S_{i}(t)
$$

( $w_{i}$ constante no tempo).

Assumimos que cada processo de preços satisfaz a seguinte relação

$$
\frac{d S_{i}}{S_{i}}(t)=\sigma_{i}\left(t, S_{i}\right) d Z_{i}+\mu_{i} d t
$$

na qual $\sigma_{i}(\cdot, \cdot)$ representa a volatilidade local do $i$-ésimo ativo, $\mu_{i}$ é o custo de carregamento, associado com o custo de manter a carteira no tempo, e 
$Z_{i} \equiv Z_{i}(t)$ é o movimento browniano padrão com a seguinte correlação

$$
d Z_{i} d Z_{j}=\rho_{i j} d t
$$

Vamos assumir que os processos $Z_{i}$ já estão ajustados ao risco e portanto assumir que $\mu_{i}=r, i=1, \ldots, n$ igual a taxa de risco do período.

Podemos supor, para obter simplicidade, que $\rho_{i j}$ é constante com respeito ao tempo, já que na verdade é a situação mais provável. Isto é porque as correlações são estimadas usando dados históricos. Embora o estudo da estimação das correlações é uma questão importante e interessante pois existem certas instabilidades, como será exposto na seção §4.1.

Um elemento importante para o nosso estudo é a variância estocástica associada ao índice. A seguir deduzimos tal fórmula.

Para o índice $B(t, \mathbf{S})=\sum_{i=1}^{n} w_{i} S_{i}(t)$ temos

$$
\begin{aligned}
d B_{t} & =\sum_{i=1}^{n} w_{i} d S_{i}(t) \\
& \text { utilizando }(1.1) \text { resulta } \\
& =\sum_{i=1}^{n} w_{i}\left(\sigma_{i}\left(t, S_{i}\right) d Z_{i}+\mu_{i} d t\right) S_{i}(t) .
\end{aligned}
$$

Logo,

$$
\begin{aligned}
\left(d B_{t}\right)^{2} & =\sum_{j, i=1}^{n} w_{j} w_{i} S_{j}(t) S_{i}(t)\left(\sigma_{j} \sigma_{i} d Z_{j} d Z_{i}+\mu_{j} \mu_{i}(d t)^{2}+2 \sigma_{j} d Z_{j} \mu_{i} d t\right) \\
& =\sum_{j, i=1}^{n} w_{j} w_{i} \rho_{i j} S_{j}(t) S_{i}(t) \sigma_{j}\left(t, S_{j}\right) \sigma_{i}\left(t, S_{i}\right) d t
\end{aligned}
$$

$\mathrm{Na}$ ultima igualdade utilizamos o fato de que o processo de preços é um processo de Itô e, portanto, $d Z_{i} d Z_{j}=\rho_{i j} d t$ e $d t d Z_{i}=d t d t=0$.

Logo a volatilidade estocástica do índice, $\sigma_{t}$, fica:

$$
\sigma_{t}^{2}(\mathbf{S})=\frac{1}{B^{2}(t, \mathbf{S})} \sum_{j, i=1}^{n} \rho_{i j} \sigma_{j}\left(t, S_{j}\right) \sigma_{i}\left(t, S_{i}\right) w_{j} w_{i} S_{j}(t) S_{i}(t) .
$$


Observemos que a expressão anterior depende dos preços dos ativos e do índice, tendo assim caráter estocástico. Como é uma função não observável, estudaremos uma média (condicionada) dela. Tal média, volatilidade local, independe de $\mathbf{S}$ e portanto será uma função determinística a qual poderemos estudar com ferramentas da análise clássica. 


\section{Capítulo 2}

\section{Reconstrução da estrutura de volatilidade do índice}

No presente capítulo definimos matematicamente a volatilidade local da carteira e aproximamos-la por uma expressão mais simples de calcular. Tal expressão envolve um estudo do comportamento assintótico de integrais e um problema de otimização. Particularmente, o método de Laplace da análise assintótica nos permite sintetizar a volatilidade local da carteira como a estocástica avaliada num ponto específico. Determinaremos tal ponto por meio da resolução de um problema de programação não linear, pois representará a configuração de preços mais provável para descrever um determinado índice.

\section{$2.1 \quad$ Volatilidade local}

Definição 2.1.1 A volatilidade local, $\sigma_{l o c}$, é uma função do índice $B \in \mathbb{R}^{+}$e do tempo $t \in \mathbb{R}_{0}^{+}$determinada pela seguinte esperança condicional

$$
\sigma_{l o c}^{2}(t, B)=\mathbb{E}\left[\sigma_{t}^{2}(\mathbf{S}) \mid B(t, \mathbf{S})=B\right] .
$$


Isto é:

$$
\sigma_{l o c}^{2}(t, B)=\mathbb{E}\left[\frac{1}{B^{2}(t, \mathbf{S})} \sum_{j, i=1}^{n} \rho_{i j} \sigma_{j} \sigma_{i} w_{j} w_{i} S_{j} S_{i} \quad \mid \sum_{i=1}^{n} w_{i} S_{i}=B\right] .
$$

A expressão acima pode ser escrita como

$$
\sigma_{l o c}^{2}(t, B)=\frac{\mathbb{E}\left[\sigma_{t}^{2}(\mathbf{S}) \delta(B(t, \mathbf{S})-B)\right]}{\mathbb{E}[\delta(B(t, \mathbf{S})-B)]}
$$

na qual $\delta(x)$ é a função delta de Dirac. De fato,

$$
\begin{aligned}
\mathbb{E}\left[\sigma_{t}^{2}(\mathbf{S}) \mid \sum_{i} w_{i} S_{i}=B\right] & =\int_{\mathbb{R}_{+}^{n}} \sigma_{t}^{2}(\mathbf{x}) \frac{f_{\mathbf{S}, B(\mathbf{S})}(\mathbf{x}, B)}{f_{B(\mathbf{S})}(B)} d \mathbf{x} \\
& =\frac{1}{f_{B(\mathbf{S})}} \int_{\mathbb{R}_{+}^{n}} \sigma_{t}^{2}(\mathbf{x}) f_{\mathbf{S}, B(\mathbf{S})}(\mathbf{x}, B) d \mathbf{x} \\
& =\frac{\int_{\mathbb{R}_{+}} \int_{\mathbb{R}_{+}^{n}} \sigma_{t}^{2}(\mathbf{x}) \delta(b-B) f_{\mathbf{S}, B(\mathbf{S})}(\mathbf{x}, B) d \mathbf{x} d b}{\int_{\mathbb{R}_{+}} \delta(b-B) f_{B(\mathbf{S})}(b) d b} \\
& =\frac{\mathbb{E}\left[\sigma_{t}^{2}(\mathbf{S}) \delta(B(t, \mathbf{S})-B)\right]}{\mathbb{E}[\delta(B(t, \mathbf{S})-B)]} .
\end{aligned}
$$

Estamos interessados em obter uma expressão mais simples de trabalhar para a equação (2.1) num instante de tempo infinitesimal, i.e., fazer um estudo para $t \downarrow 0$. Com este fim aplicamos a seguinte mudança de variáveis

$$
x_{i}(t)=\ln \left(\frac{S_{i}(t)}{S_{i}(0) e^{\mu_{i} t}}\right) \equiv \ln \left(\frac{S_{i}(t)}{F_{i}(t)}\right) .
$$

Ou seja, trabalharemos com o processo dos logaritmos dos preços descontados, já que, supondo que $\mu_{i}$ representa a taxa livre de risco associada ao $i$-ésimo ativo, $F_{i}(t) \equiv S_{i}(0) e^{\mu_{i} t}$ é o valor futuro do ativo. A mudança de variáveis para os logaritmos definida por (2.2) é muito utilizada em finanças, pois permite ter uma melhor noção do retorno do ativo. 
Aplicando o lema de Itô às variáveis $x_{i}$ definidas em (2.2), obtemos o seguinte processo

$$
d \mathbf{x}_{t}=\left[\begin{array}{c}
\mu_{1} \\
\cdot \\
\mu_{n}
\end{array}\right] d t+A \cdot\left[\begin{array}{c}
d Z_{1} \\
\vdots \\
d Z_{n}
\end{array}\right]
$$

em que $A$ é a matriz de coeficientes de difusão do processo $\mathrm{x}_{t}$ definida por $a^{i j}=\sigma_{i}\left(t, x_{i}\right) \sigma_{j}\left(t, x_{j}\right) \rho_{i j}$.

Isto é, para todo $i=1, \ldots, n$

$$
d x_{i}=\mu_{i} d t+\sum_{j=1}^{n} \sigma_{i}\left(t, x_{i}\right) \sigma_{j}\left(t, x_{j}\right) \rho_{i j} d Z_{j} .
$$

A matriz de coeficientes de difusão $A$ é a matriz de covariância do processo $\mathrm{x}_{t} \mathrm{e}$, portanto, é simétrica. Vamos assumir também por hipótese, que os ativos não são redundantes, isto é, o retorno de nenhum ativo é explicado pelos retornos dos outros ( ou seja, os $x_{i}$ são linearmente independentes). Deste fato, segue que $A$ é inversível. Assim, denotamos por $\left(g_{i j}\right)$ a matriz inversa de $A$ e consideremos a métrica Riemanniana associada:

$$
d s^{2}=\sum_{i, j=1}^{n} g_{i j} d x_{i} d x_{j}
$$

Logo a probabilidade de transição do processo $\mathrm{x}_{t}, p\left(t_{0}, \mathrm{x}_{0} ; t, \mathrm{x}\right)$, está dada pela solução fundamental do seguinte problema de Cauchy para a equação de Kolmogorov (ver [Øks00]):

$$
\left\{\begin{array}{l}
\frac{\partial p}{\partial t}=\frac{1}{2} \sum_{i, j=1}^{n} a^{i j} \frac{\partial^{2} p}{\partial x_{i} \partial x_{j}} \\
p\left(t_{0}, \mathrm{x}_{0} ; 0, \mathrm{x}\right)=\delta_{\mathrm{x}_{0}}(\mathrm{x})
\end{array}\right.
$$

Ou seja, $p$ mede a probabilidade de passar do estado $\mathrm{x}_{0}$ no tempo $t_{0}$ para um estado suficientemente próximo de $\mathrm{x}$, é dizer $|\mathrm{x}(t)-\mathrm{x}|<|d \mathrm{x}|$, num intervalo de tempo $t-t_{0}$, isto é:

$$
\left.p\left(t_{0}, \mathrm{x}_{0} ; t, \mathrm{x}\right) d \mathrm{x}=\mathbf{P}\{\mathrm{x}(t):|\mathrm{x}(t)-\mathrm{x}|<|d \mathrm{x}|) \mid \mathrm{x}\left(t_{0}\right)=\mathrm{x}_{0}\right\} .
$$


Durante o trabalho todo, para simplificar, permitimos o seguinte abuso de notação, explicitando apenas a dependência em x:

$$
\begin{aligned}
B(t, \mathrm{x}) & \equiv B\left(t,\left(F_{1}(t) e^{x_{1}}, \cdots, F_{n}(t) e^{x_{n}}\right)\right) \\
& =\sum_{i=1}^{n} w_{i} F_{i}(t) e^{x_{i}} \\
\sigma_{t}^{2}(\mathrm{x}) & \equiv \frac{1}{B^{2}(t, \mathrm{x})} \sum_{j, i=1}^{n} \rho_{i j} \sigma_{j}\left(t, x_{j}\right) \sigma_{i}\left(t, x_{i}\right) w_{j} w_{i} F_{j}(t) F_{i}(t) e^{x_{j}} e^{x_{j}}, \\
\sigma_{i}\left(t, x_{i}\right) & \equiv \sigma_{i}\left(t, F_{i}(t) e^{x_{i}}\right) \forall i=1, \ldots, n .
\end{aligned}
$$

Com tal notação, temos:

$$
\begin{aligned}
\sigma_{l o c}^{2}(t, B) & =\frac{1}{\mathbb{E}[\delta(B(t, \mathbf{x})-B)]} \int_{\mathbb{R}^{n}} \sigma_{t}^{2}(\mathbf{x}) \delta(B(t, \mathbf{x})-B) p(0, \mathbf{0} ; t, \mathbf{x}) d \mathbf{x} \\
& =\frac{1}{\left\|\Gamma_{B}(t)\right\|_{p}} \int_{\mathbb{R}^{n}} \sigma_{t}^{2}(\mathbf{x}) \delta(B(t, \mathbf{x})-B) p(0, \mathbf{0} ; t, \mathbf{x}) d \mathbf{x}
\end{aligned}
$$

em que $\Gamma_{B}(t) \doteq\left\{\mathrm{x} \in \mathbb{R}^{n}: B(t, \mathrm{x})=B\right\}$ e $\left\|\Gamma_{B}(t)\right\|_{p}=\mathbb{E}[\delta(B(t, \mathrm{x})-B)]$. A grandeza $\left\|\Gamma_{B}(t)\right\|_{p}$ pode ser interpretada como o volume médio de $\Gamma_{B}(t)$ ponderada por $p$. Por outro lado, podemos ver que $\Gamma_{B}(t)$ é não vazia pois para cada $t \geq 0$, o ponto $\mathbf{z}^{t} \in \mathbb{R}^{n}$ definido por

$$
\mathrm{z}^{t}=\left(\frac{1}{n} \ln \left(\frac{B}{w_{1} F_{1}(t)}\right), \cdots, \frac{1}{n} \ln \left(\frac{B}{w_{n} F_{n}(t)}\right)\right),
$$

trivialmente está em $\Gamma_{B}(t)$.

O cálculo da integral

$$
\sigma_{l o c}^{2}(t, B)=\frac{1}{\left\|\Gamma_{B}(t)\right\|_{p}} \int_{\mathbb{R}^{n}} \sigma_{t}^{2}(\mathbf{x}) \delta(B(t, \mathbf{x})-B) p(0, \mathbf{0} ; t, \mathbf{x}) d \mathbf{x}
$$

poderia ser feito diretamente se conhecêssemos a solução fundamental $p$ do problema (2.4), uma tarefa bastante difícil de ser realizada. Como estamos interessados na síntese da estrutura de volatilidade do índice $B$ para $t=0$, o cálculo assintótico da solução em uma variedade Riemanniana, quando $t \downarrow 0$, é no entanto mais simples e é suficiente para nossos propósitos. Este cálculo 
assintótico foi feito por Varadhan [Var67b] e [Var67a], obtendo a seguinte fórmula

$$
\lim _{t \rightarrow 0^{+}}[-2 t \log p(0,0 ; t, \mathbf{x})]=d_{g}^{2}(\mathbf{0}, \mathbf{x}) \quad \text { uniformemente em } \mathbf{x},
$$

na qual

$$
d_{g}^{2}(0, \mathrm{x})=\inf _{\mathrm{x}(0)=\mathbf{0}, \mathrm{x}(1)=\mathrm{x}} \int_{0}^{1} \sum_{i, j=1}^{n} g_{i j}(\mathrm{x}(s), 0) \dot{x}_{i} \dot{x}_{j} d s .
$$

Equivalentemente, temos que:

$$
-2 t \log p(0, \mathbf{0} ; t, \mathrm{x})=d_{g}^{2}(\mathbf{0}, \mathrm{x})+r(t, \mathrm{x})
$$

e portanto:

$$
p(0, \mathbf{0} ; t, \mathbf{x})=\exp \left(-\frac{d_{g}^{2}(\mathbf{0}, \mathbf{x})}{2 t}-\frac{r(t, \mathrm{x})}{2 t}\right)
$$

onde $|r(t, \mathrm{x})| \stackrel{t\rfloor 0}{\longrightarrow} 0$ uniformemente em $\mathbf{x}$.

Portanto, a volatilidade local está dada por:

$$
\sigma_{l o c}^{2}(t, B)=\frac{1}{\left\|\Gamma_{B}(t)\right\|_{p}} \int_{\mathbb{R}^{n}} \sigma_{t}^{2}(\mathrm{x}) \delta(B(t, \mathbf{x})-B) e^{-\frac{d_{g}^{2}(0, \mathbf{x})-r(t, \mathbf{x})}{2 t}} d \mathbf{x} .
$$

No contexto da fórmula de Varadhan, a distância é equivalente à norma euclidiana, isto é, existem constantes $C_{1}>0$ e $C_{2}>0$ tais que

$$
C_{1}|\mathrm{x}-\mathrm{y}| \leq d_{g}^{2}(\mathrm{y}, \mathrm{x}) \leq C_{2}|\mathrm{x}-\mathrm{y}|
$$

A expressão (2.7) vai nos permitir obter uma fórmula fechada para aproximar a volatilidade local da carteira dado um índice $B$. A idéia central consiste em calcular a integral somente sobre vizinhanças de pontos que minimizam a distância à origem, pois a exponencial negativa faz que a massa total esteja concentrada naqueles pontos.

Durante o trabalho todo, vamos supor que $\forall i=1, \ldots, n$ existem $0<\sigma_{\min }^{i} \leq\left|\sigma_{i}\left(t, x_{i}\right)\right| \leq \sigma_{\max }^{i}$. Com isto podemos afirmar que

$$
\left|\sigma_{t}^{2}(\mathrm{x})\right|<e^{|\mathrm{x}|} \text {, uniformemente em } t
$$


É razoável supor que as volatilidades locais dos ativos sejam limitadas, já que, pela história do mercado, sabemos que tais volatilidades não podem tomar valores indefinidamente pequenos e tampouco indefinidamente grandes.

\subsection{Aproximação assintótica}

Em virtude da expansão assintótica dada pela fórmula de Varadhan, podemos utilizar o método de Laplace (apresentado no apêndice) para o cálculo das integrais que definem a volatilidade local. Aplicamos tal método para um ponto minimizador da distância, já que, localmente, a distância é uma função quadrática.

Pelas propriedades de $\Gamma_{B}(t)$ e da métrica $d_{g}^{2}$, podemos supor que temos um único ponto minimizador $\mathrm{x}^{*},{ }^{1}$ esquecendo das simetrias e pontos isolados. Tais situações se apresentam em ocasiões muito peculiares do mercado, sendo pouco prováveis. É dizer, se em algum instante temos no mercado varias cotações dos ativos da carteira que maximizam a probabilidade de reproduzir o índice, imediatamente esta simetria será quebrada pela aleatoriedade dos preços.

Expressamos o erro $r(t, \mathrm{x})$ sob a forma $e^{-\frac{r(t, \mathrm{x})}{2 t}}=[1+\Upsilon(t, \mathrm{x})]$, onde $\Upsilon(t, \mathrm{x})=o(1)$ uniformemente em $t \downarrow 0$. Assim, obtemos a expressão:

$$
\sigma_{\text {loc }}^{2}(t, B)=\frac{1}{\left\|\Gamma_{B}(t)\right\|_{p}}\left[\int_{\mathbb{R}^{n}} \sigma_{t}^{2}(\mathrm{x}) \delta(B(t, \mathrm{x})-B) e^{-\frac{d_{g}^{2}(0, \mathrm{x})}{2 t}} d \mathbf{x}+\text { erro }\right]
$$

O esquema que pretendemos fazer está dado pela seguinte figura, na qual, dentro da região $B_{\delta^{*}}$ está acumulada a maior massa da integral e fora temos uma expressão infinitesimal quando $t \downarrow 0$; assim, a integral (2.10) pode ser aproximada pela função em $\mathrm{x}^{*}$.

\footnotetext{
${ }^{1}$ Se tivermos vários minimizadores para aplicar o método de Laplace, simplesmente partimos a integral em integrais sobre regiões nas quais a função tem um minimizador só (ver $\S 2[$ Mur84]).
} 


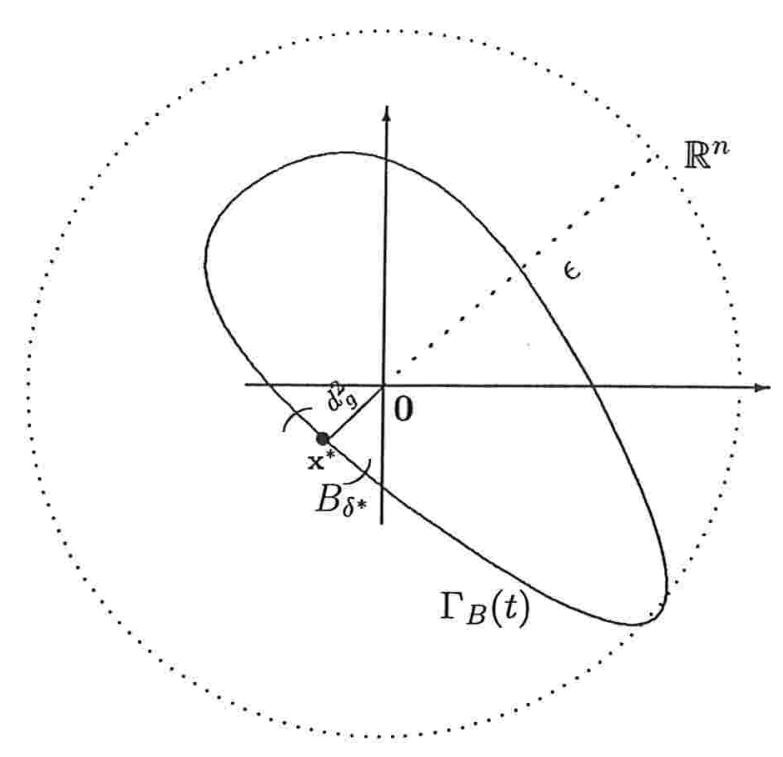

Observemos que no esquema anterior desenhamos uma bola de raio $\epsilon$ para ressaltar que nossos cálculos serão feitos localmente, estando sempre em pequenas vizinhanças da origem.

Seja $\mathrm{x}^{*} \in \mathbb{R}^{n}$ a solução do seguinte problema de minimização

$$
\begin{cases}\min _{\mathbf{x}} & d_{g}^{2}(0, \mathrm{x}) \\ \text { s.a. } & \mathrm{x} \in \Gamma_{B}(t)\end{cases}
$$

Localmente a distância é uma forma quadrática, isto é, existe $\delta\left(\mathrm{x}^{*}\right) \equiv \delta^{*}$ tal que $\forall \mathrm{x}:\left|\mathrm{x}-\mathrm{x}^{*}\right|<\delta^{*}$

$$
d_{g}^{2}(0, \mathrm{x})=d_{g}^{2}\left(0, \mathrm{x}^{*}\right)+\left.\frac{1}{2} \sum_{i, j=1}^{n} \frac{\partial^{2} d_{g}^{2}(0, \mathrm{x})}{\partial x_{i} \partial x_{j}}\right|_{\mathrm{x}=\mathrm{x}^{*}} x_{i} x_{j}+O\left(|\mathrm{x}|^{3}\right) .
$$

Não temos termo de ordem linear pois sendo $\mathrm{x}^{*}$ o minimizador, $\nabla d_{g}^{2}(0, \mathrm{x})\left(\mathrm{x}^{*}\right) \equiv \mathbf{0}$.

Portanto, aplicando este desenvolvimento de segunda ordem na equação (2.7), temos

$$
\sigma_{l o c}^{2}(t, B)=\frac{1}{\left\|\Gamma_{B}(t)\right\|_{p}}\{I+I I+\text { erro }\}
$$


onde

$$
\begin{aligned}
I & =\int_{\mathcal{U}_{\delta^{*}}} \sigma_{t}^{2}(\mathrm{x}) \delta(B(t, \mathrm{x})-B) e^{-\frac{1}{2 t}\left[d_{g}^{2}\left(0, \mathbf{x}^{*}\right)+\left.\frac{1}{2} \sum_{i, j} \frac{\partial^{2} d_{g}^{2}(0, \mathbf{x})}{\partial x_{i} \partial x_{j}}\right|_{\mathbf{x}=\mathbf{x}^{*}} x_{i} x_{j}\right]} d \mathrm{x} \\
I I & =\int_{\mathbb{R}^{n} \backslash \mathcal{U}_{\delta^{*}}} \sigma_{t}^{2}(\mathrm{x}) \delta(B(t, \mathrm{x})-B) e^{\frac{-d^{2}(\mathbf{0}, \mathbf{x})}{2 t}} d \mathrm{x},
\end{aligned}
$$

nas quais $\mathcal{U}_{\delta^{*}} \equiv\left\{\mathrm{x} \in \mathbb{R}^{n}:\left|\mathrm{x}-\mathrm{x}^{*}\right|<\delta^{*}\right\}$.

Vamos aplicar o método de Laplace à integral $I$. Com tal fim, definimos a seguinte mudança de variáveis

$$
|\mathrm{y}|^{2}=\left.\frac{1}{2} \sum_{i, j=1}^{n} \frac{\partial^{2} d_{g}^{2}(0, \mathbf{x})}{\partial x_{i} \partial x_{j}}\right|_{\mathbf{x}=\mathbf{x}^{*}} x_{i} x_{j}
$$

isto é, $\mathrm{y}=\frac{1}{\sqrt{2}} Q \mathrm{x}$ onde $\left(\left.\frac{\partial^{2} d_{g}^{2}(0, \mathrm{x})}{\partial x_{i} \partial x_{j}}\right|_{\mathbf{x}=\mathbf{x}^{*}}\right)_{i j}=Q^{T} Q$. Assim, obtemos a integral

$$
\left.I=e^{-\frac{d_{g}^{2}\left(\mathbf{0}, \mathbf{x}^{*}\right)}{2 t}} \int_{\mathcal{U}_{\delta^{*}}} \sigma_{t}^{2}(\mathrm{x}(\mathrm{y})) \delta(B(t, \mathbf{x}(\mathbf{y}))-B)\left|J_{t}(\mathbf{y})\right|^{-1} e^{\frac{-1}{2 t}}|\mathbf{y}|^{2}\right) d \mathbf{y}
$$

na qual $\left|J_{t}(\mathrm{y})\right|$ representa o determinante da matriz jacobiana da mudança de variáveis (2.11). Agora podemos aplicar o método de Laplace, obtendo assim

$$
I=\sigma_{t}^{2}\left(\mathrm{x}^{*}\right)(2 t \pi)^{n / 2} e^{-\frac{d_{g}^{2}\left(\mathbf{0}, \mathbf{x}^{*}\right)}{2 t}}\left|J_{t}\left(\mathrm{x}^{*}\right)\right|+O\left(t^{n / 2+1}\right) .
$$

Por outro lado, analisamos a integral $I I$. Para isto utilizamos as propriedades (2.8) e (2.9).

Usando (2.8), temos que $|\mathrm{x}| \leq \frac{1}{C_{1}} d_{g}^{2}(0, \mathrm{x})$ e $\forall \mathrm{x} \in \mathbb{R}^{n} \backslash \mathcal{U}_{\delta^{*}}$ temos que:

$$
\begin{aligned}
\delta^{*} \leq\left|\mathrm{x}-\mathrm{x}^{*}\right| & \leq \frac{1}{C_{1}} d_{g}^{2}\left(\mathrm{x}, \mathrm{x}^{*}\right) \\
& \leq \frac{1}{C_{1}}\left(d_{g}^{2}\left(0, \mathrm{x}^{*}\right)-d_{g}^{2}(0, \mathrm{x})\right) \\
& \leq \frac{2}{C_{1}} d_{g}^{2}(0, \mathrm{x}) \quad \text { pois } \mathrm{x}^{*} \text { é o minimizador } \\
& \therefore 0<\frac{\delta^{*} C_{1}}{2} \leq d_{g}^{2}(0, \mathrm{x}) .
\end{aligned}
$$


Aplicando os resultados anteriores e a propriedade (2.9) na integral $I I$, obtemos

$$
\begin{aligned}
I I & =\int_{\mathbb{R}^{n} \backslash \mathcal{U}_{\delta^{*}}} \sigma_{t}^{2}(\mathrm{x}) \delta(B(t, \mathrm{x}-B)) e^{-\frac{d_{g}^{2}(0, \mathrm{x})}{2 t}} d \mathrm{x} \\
& \leq \int_{\mathbb{R}^{n} \backslash \mathcal{U}_{\delta^{*}}} \delta(B(t, \mathrm{x}-B)) e^{|\mathbf{x}|} e^{-\frac{d_{g}^{2}(0, \mathrm{x})}{2 t}} d \mathrm{x} \\
& \leq \int_{\mathbb{R}^{n} \backslash \mathcal{U}_{\delta^{*}}} \delta(B(t, \mathrm{x}-B)) e^{\left(\frac{1}{C_{1}}-\frac{1}{2 t}\right) d_{g}^{2}(0, \mathrm{x})} d \mathrm{x} \\
& \leq e^{\left(\operatorname{para} t \ll 1 \operatorname{temos} \frac{1}{C_{1}}-\frac{1}{2 t}\right) \frac{\delta^{*} C_{1}}{2}} \int_{\mathbb{R}^{n} \backslash \mathcal{U}_{\delta^{*}}} \delta(B(t, \mathrm{x}-B)) d \mathrm{x} \\
& =\operatorname{Vol}\left(\Gamma_{B}(t) \backslash \mathcal{U}_{\delta^{*}}\right) e^{\left(\frac{1}{C_{1}}-\frac{1}{2 t}\right) \frac{\delta^{*} C_{1}}{2}} \\
& =O\left(t^{n / 2+1}\right),
\end{aligned}
$$

pois o termo exponencial em $t$ é dominado por um polinômio de qualquer grau.

Portanto podemos afirmar que:

$$
\sigma_{l o c}^{2}(t, B)=\frac{\sigma_{t}^{2}\left(\mathrm{x}^{*}\right)(2 t \pi)^{n / 2} e^{-\frac{d_{g}^{2}\left(\mathbf{0}, \mathrm{x}^{*}\right)}{2 t}}\left|J_{t}\left(\mathrm{x}^{*}\right)\right|+O\left(t^{n / 2+1}\right)+\text { erro }}{\left\|\Gamma_{B}(t)\right\|_{p}} .
$$

Por meio de um procedimento semelhante, obtemos:

$$
\left\|\Gamma_{B}(t)\right\|_{p}=(2 t \pi)^{n / 2} e^{-\frac{d_{g}^{2}\left(0, \mathbf{x}^{*}\right)}{2 t}}\left|J_{t}\left(\mathrm{x}^{*}\right)\right|+O\left(t^{n / 2+1}\right)+\text { erro. }
$$

Das equações (2.14) e (2.15), dado que $\sigma_{t}^{2}\left(\mathrm{x}^{*}\right)$ é limitada, obtemos a seguinte expressão assintótica para a volatilidade local:

$$
\sigma_{l o c}^{2}(t, B)=\sigma_{t}^{2}\left(\mathrm{x}^{*}\right)\left[1+\Upsilon\left(t, \mathrm{x}^{*}\right)\right], \quad \Upsilon\left(t, \mathrm{x}^{*}\right) \stackrel{t \downarrow 0}{\longrightarrow} 0 .
$$

Logo, temos uma nova questão que é determinar $\mathrm{x}^{*}$, esta é desenvolvida na seguinte seção. 


\subsection{A configuração mais provável de atingir o ín- dice}

Nesta seção procuramos uma expressão para o minimizador $x^{*}$. Lembremos que da fórmula de Varadhan deduzimos que:

$$
p(0,0 ; t, \mathrm{x}) \approx \exp \left(-\frac{d_{g}^{2}(0, \mathrm{x})}{2 t}\right)
$$

$\mathrm{e}$, portanto, caracterizando tal minimizador para $t \ll 1$ obtemos a configuração $\mathbf{S}^{*}$ de preços que é mais provável de atingir o índice $B$. Assim, conseguimos aproximar a volatilidade local da carteira para esse índice.

Para representar a porcentagem do ativo $i$ no índice para o instante $t$, estabelecemos a seguinte notação

$$
p_{i}(t, \mathrm{x}) \equiv \frac{F_{i}(t) e^{x_{i}} w_{i}}{B(t, \mathrm{x})}=\frac{F_{i}(t) e^{x_{i}} w_{i}}{\sum_{j=1}^{n} F_{j}(t) e^{x_{j}} w_{j}}
$$

lembrando que $S_{i}(t)=F_{i}(t) e^{x_{i}}$.

Observando que a métrica satisfaz

$$
\sum_{i, j=1}^{n} g_{i j}(0, \mathrm{x}) d x_{i} d x_{j}=\sum_{i, j=1}^{n}\left(\rho^{-1}\right)_{i j} \frac{d x_{i}}{\sigma_{i}\left(t, x_{i}\right)} \frac{d x_{j}}{\sigma_{j}\left(t, x_{j}\right)},
$$

pois

$$
\begin{aligned}
\sum_{i, j=1}^{n} g_{i j}(\mathbf{0}, \mathbf{x}) d x_{i} d x_{j} & =\dot{\mathrm{x}}^{T} a^{-1} \dot{\mathrm{x}} \\
& =\dot{\mathrm{x}}^{T}\left(\begin{array}{ccc}
\frac{1}{\sigma_{1}} & & \\
0 & \ddots & 0 \\
& & \frac{1}{\sigma_{n}}
\end{array}\right) \rho^{-1}\left(\begin{array}{ccc}
\frac{1}{\sigma_{1}} & & \\
0 & \ddots & 0 \\
& & \frac{1}{\sigma_{n}}
\end{array}\right) \dot{\mathrm{x}} \\
& =\sum_{i, j=1}^{n}\left(\rho^{-1}\right)_{i j} \frac{d x_{i}}{\sigma_{i}\left(t, x_{i}\right)} \frac{d x_{j}}{\sigma_{j}\left(t, x_{j}\right)} .
\end{aligned}
$$

O problema de otimização será resolvido por meio de uma mudança de variáveis que nos permitirá simplificar a expressão da distância. Tal 
mudança é definida por:

$$
y_{i}=\int_{0}^{x_{i}} \frac{d u}{\sigma_{i}(t, u)}
$$

em que $d y_{i}=\frac{d x_{i}}{\sigma_{i}\left(t, x_{i}\right)}$, assim nosso problema de minimização resulta ser

$$
\begin{cases}\min _{\mathrm{y}} & \int_{0}^{1} \sum_{i, j=1}^{n}\left(\rho^{-1}\right)_{i j} \dot{y}_{i} \dot{y}_{j} d s \\ \text { s.a. } & \mathrm{x}(\mathrm{y}) \in \Gamma_{B}(t)\end{cases}
$$

Como o problema anterior é de otimização restrita não linear, empregaremos o Lagrangeano. Aplicando as condições de primeira ordem de tal método ( $\$ 10$ [Lue84]) obtemos que o problema de minimização anterior é equivalente a resolver o seguinte sistema de equações em y e $\tilde{\lambda}$

$$
\begin{aligned}
\nabla\left(\int_{0}^{1} \sum_{i, j=1}^{n}\left(\rho^{-1}\right)_{i j} \dot{y}_{i} \dot{y}_{j} d s\right)+\tilde{\lambda} \nabla\left(B-\sum_{i=1}^{n} w_{i} F_{i}(t) e^{x_{i}\left(y_{i}\right)}\right) & =0 \\
\sum_{i=1}^{n} w_{i} F_{i}(t) e^{x_{i}\left(y_{i}\right)} & =B,
\end{aligned}
$$

em que $\tilde{\lambda}$ é o multiplicador de Lagrange associado à restrição $\mathrm{x}(\mathrm{y}) \in \Gamma_{B}(t)$ equivalente com $B-\sum_{i=1}^{n} w_{i} F_{i}(t) e^{x_{i}\left(y_{i}\right)}=0$.

Como $d y_{i}$ é constante, é igual a sua média no intervalo $(0,1)$ e assim temos $d y_{i}=y_{i}, i=1, \ldots, n$. Logo, para toda coordenada $k=1, \ldots, n$, a igualdade (2.20) fica:

$$
\frac{\partial}{\partial y_{k}} \int_{0}^{1} \sum_{i=1}^{n} \sum_{j=1}^{n}\left(\rho^{-1}\right)_{i j} y_{i} y_{j} d s=\tilde{\lambda} \frac{\partial}{\partial y_{k}}\left(-B+\sum_{i=1}^{n} w_{i} F_{i}(t) e^{x_{i}\left(y_{i}\right)}\right) .
$$

Utilizando que $y_{k}$ é constante no primeiro somando e derivando em relação à $y_{k}$ no segundo, temos que:

$$
\frac{\partial}{\partial y_{k}} \sum_{i=1}^{n} \sum_{j=1}^{n}\left(\rho^{-1}\right)_{i j} y_{i} y_{j} \int_{0}^{1} d s=\tilde{\lambda} \sum_{i=1}^{n} w_{i} F_{i}(t) e^{x_{i}} \frac{\partial x_{i}\left(y_{i}\right)}{\partial y_{k}} .
$$


Tomando $k=i$, obtemos:

$$
\begin{aligned}
2 \sum_{j=1}^{n}\left(\rho^{-1}\right)_{i j} y_{j} & =\tilde{\lambda} w_{i} F_{i}(t) e^{x_{i}}\left(\frac{\partial y_{i}\left(x_{i}\right)}{\partial x_{i}}\right)^{-1} \\
& =\tilde{\lambda} w_{i} F_{i}(t) e^{x_{i}} \sigma_{i}\left(t, x_{i}\right) .
\end{aligned}
$$

Logo a equação (2.22) pode ser escrita matricialmente como:

$$
\rho^{-1} \cdot \mathrm{y}=\frac{1}{2}\left[\begin{array}{c}
\tilde{\lambda} B(t, \mathrm{x}) \frac{w_{1} F_{1}(t) e^{x_{1}} \sigma_{1}\left(t, x_{1}\right)}{B(t, \mathbf{x})} \\
\vdots \\
\tilde{\lambda} B(t, \mathbf{x}) \frac{w_{n} F_{n}(t) e^{x_{n}} \sigma_{n}\left(t, x_{n}\right)}{B(t, \mathbf{x})}
\end{array}\right]
$$

Aplicando $\rho$ em ambos os termos, temos:

$$
\begin{aligned}
& \mathbf{y}=\frac{1}{2} \rho \cdot\left[\begin{array}{c}
\tilde{\lambda} B(t, \mathrm{x}) \frac{w_{1} F_{1}(t) e^{x_{1} \sigma_{1}\left(t, x_{1}\right)}}{B(t, \mathbf{x})} \\
\vdots \\
\tilde{\lambda} B(t, \mathrm{x}) \frac{w_{n} F_{n}(t) e^{x_{n}} \sigma_{n}\left(t, x_{n}\right)}{B(t, \mathbf{x})}
\end{array}\right] \\
& \mathbf{y}=\frac{1}{2}\left[\begin{array}{c}
\tilde{\lambda} B(t, \mathrm{x}) \sum_{j=1}^{n} p_{j}(t, \mathrm{x}) \rho_{1 j} \sigma_{1}\left(t, x_{j}\right) \\
\vdots \\
\tilde{\lambda} B(t, \mathrm{x}) \sum_{j=1}^{n} p_{j}(t, \mathrm{x}) \rho_{n j} \sigma_{j}\left(t, x_{j}\right)
\end{array}\right]
\end{aligned}
$$

Substituindo na equação (2.23), obtemos:

$$
\int_{0}^{x_{i}} \frac{d u}{\sigma_{i}(t, u)}=\frac{\tilde{\lambda}}{2} B(t, \mathrm{x}) \sum_{j=1}^{n} p_{j}(t, \mathrm{x}) \rho_{i j} \sigma_{j}\left(t, x_{j}\right), \quad \forall i=1, \ldots, n
$$

Para simplificar, reescrevemos o multiplicador de Lagrange $\tilde{\lambda}$ como $\lambda / B$ e então a equação (2.24), utilizando a condição $B(t, \mathrm{x})=B$, fica:

$$
\int_{0}^{x_{i}} \frac{d u}{\sigma_{i}(t, u)}=\frac{\lambda}{2} \sum_{j=1}^{n} p_{j}(t, \mathrm{x}) \rho_{i j} \sigma_{j}\left(t, x_{j}\right), \quad \forall i=1, \ldots, n
$$

Com isto, resolvemos o nosso problema de otimização (2.19), obtendo assim, para $t \ll 1$, a volatilidade local do $B$ : 


$$
\sigma_{l o c}^{2}(t, B)=\left[1+\Upsilon\left(t, \mathrm{x}^{*}\right)\right] \sum_{i, j=1}^{n} \rho_{i j} \sigma_{i}\left(t, x_{i}^{*}\right) \sigma_{j}\left(t, x_{j}^{*}\right) p_{i}\left(t, \mathrm{x}^{*}\right) p_{j}\left(t, \mathrm{x}^{*}\right) .
$$

na qual $x^{*}$ é a solução do seguinte sistema:

$$
\left\{\begin{array}{l}
\int_{0}^{x_{i}^{*}} \frac{d u}{\sigma_{i}(t, u)}=\frac{\lambda}{2} \sum_{j=1}^{n} p_{j}\left(t, \mathrm{x}^{*}\right) \rho_{i j} \sigma_{j}\left(t, x_{j}^{*}\right), \quad \forall i=1, \ldots, n \\
\sum_{i=1}^{n} w_{i} F_{i}(t) e^{x_{i}^{*}}=B .
\end{array}\right.
$$

O sistema anterior é um sistema de equações não lineares em $(\mathrm{x}, \lambda) \in \mathbb{R}^{n} \times \mathbb{R}$ cuja solução pode ser obtida por métodos numéricos como linearização das equações. Com tal fim, consideramos a seguinte expansão linear

$$
\frac{\lambda}{2} \sum_{j=1}^{n} \rho_{i j} p_{j}\left(t, \mathrm{x}^{*}\right) \sigma_{j}\left(t, x_{j}^{*}\right)=\frac{\lambda}{2} \sum_{j=1}^{n} \rho_{i j} p_{j}(t, 0) \sigma_{j}(t, 0)+O(|\mathrm{x}|) \quad \forall i=1, \ldots, n .
$$

Por outro lado, utilizando que $\left|x_{i}^{*}\right| \rightarrow 0$ para todo $i=1, \ldots, n$, temos que:

$$
\begin{aligned}
\int_{0}^{x_{i}^{*}} \frac{d u}{\sigma_{i}(t, u)} & =\frac{x_{i}^{*}}{2}\left(\frac{1}{\sigma_{i}(t, 0)}+\frac{1}{\sigma_{i}\left(t, x_{i}\right)}\right)+O\left(x_{i}^{*^{2}}\right) \\
\int_{0}^{x_{i}^{*}} \frac{d u}{\sigma_{i}(t, u)} & =\frac{x_{i}^{*}}{2 \sigma_{i}(t, 0)}+O\left(x_{i}\right) .
\end{aligned}
$$

Logo, pelas equações (2.27), (2.28) e (2.30) obtemos:

$$
x_{i}^{*}=\lambda \sigma_{i}(t, 0) \sum_{j=1}^{n} \rho_{i j} p_{j}(t, 0) \sigma_{j}(t, 0)+O(|\mathrm{x}|) \quad \forall i=1, \ldots, n .
$$

Portanto

$$
\sum_{i=1}^{n} p_{i}(t, 0) x_{i}^{*}=\lambda \sigma_{t}^{2}(0)+O(|\mathrm{x}|)
$$


Por outro lado, assim como aplicamos para os preços $S_{i}$ a mudança de variáveis $x_{i}$, podemos aplicar uma mudança análoga para os índices da carteira. Tal mudança nos $S_{i}$ é interpretada financeiramente como os logaritmos dos preços descontados. Portanto, sabendo que o valor futuro da carteira é $B(t, 0) e^{\mu_{B} t}$, definimos

$$
\bar{x} \doteq \ln \left(\frac{B}{B(t, 0) e^{\mu_{B} t}}\right),
$$

onde $\mu_{B}$ é a taxa de retorno associada à carteira.

Como $\bar{x}$ está associada com o valor descontado do índice, é natural pensar que é aproximadamente uma média ponderada dos valores $x_{i}^{*}$ associados com os preços descontados que reproduzem tal índice, é dizer,

$$
B(t, 0) e^{\bar{x}}=B=\sum_{i=1}^{n} w_{i} F_{i}(t) e^{x_{i}^{*}}
$$

Dividindo por $B(t, 0)$ e tomando logaritmos fica:

$$
\bar{x}=\log \left(\sum_{i=1}^{n} p_{i}(t, \mathbf{0}) e^{x_{i}^{*}}\right),
$$

expandindo em série de Taylor para $\mathrm{x}^{*}=\mathbf{0}$ :

$$
\begin{aligned}
\bar{x} & =\log \left(\sum_{i=1}^{n} p_{i}(t, 0)\left(1+x_{i}^{*}+O\left(x_{i}^{*^{2}}\right)\right)\right) \\
& =\log \left(1+\sum_{i=1}^{n} p(t, 0) x_{i}^{*}+O\left(x_{i}^{*^{2}}\right)\right),
\end{aligned}
$$

lembrando que $\sum_{i=1}^{n} p_{i}(t, 0)=1$.

Da equação (2.34) e do fato de que $\log (1+x)=x+O\left(|x|^{2}\right)$ para $|x| \rightarrow 0$ obtemos a aproximação:

$$
\bar{x}=\sum_{i=1}^{n} p_{i}(t, 0) x_{i}^{*}+O\left(|\mathrm{x}|^{2}\right) .
$$

Aplicando estas aproximações nas equações (2.32) e (2.31) obtemos uma caracterização da configuração dos preços mais prováveis de atingir o índice 
$B$

$$
\left\{\begin{array}{l}
x_{i}^{*}=\lambda \sum_{j=1}^{n} \rho_{i j} p_{j}(t, 0) \sigma_{j}(t, 0)+O(|\mathrm{x}|) \quad \forall i=1, \ldots, n \\
\lambda=\frac{\bar{x}}{\sigma_{t}^{2}(0)}+O(|\mathbf{x}|) .
\end{array}\right.
$$

A seguir estudamos o comportamento das expressões da volatilidade local e do minimizador no instante $t=0$. É dizer, queremos obter $\sigma_{l o c}^{2}(0, B)$ em função de dados do instante 0 , pois issos são os dados que podemos observar no mercado.

As fórmulas obtidas são:

$$
\begin{aligned}
\sigma_{l o c}^{2}(t, B) & =\sigma_{t}^{2}\left(\mathrm{x}^{*}\right)\left[1+\Upsilon\left(t, \mathrm{x}^{*}\right)\right] \\
x_{i}^{*} & =\lambda \sum_{j=1}^{n} \rho_{i j} p_{j}(t, 0) \sigma_{j}(t, 0)+O(|\mathrm{x}|) \quad \forall i=1, \ldots, n \\
\lambda & =\frac{\bar{x}}{\sigma_{t}^{2}(\mathbf{0})}+O(|\mathrm{x}|) .
\end{aligned}
$$

Calculando para cada uma o limite quando $t \downarrow 0$, obtemos:

$$
\begin{aligned}
\sigma_{l o c}^{2}(0, B) & =\sigma_{0}^{2}\left(\mathrm{x}^{*}\right) \\
x_{i}^{*} & =\lambda \sum_{j=1}^{n} \rho_{i j} p_{j}(0,0) \sigma_{j}(0,0) \quad \forall i=1, \ldots, n \\
\lambda & =\frac{\bar{x}}{\sigma_{0}^{2}(\mathbf{0})} .
\end{aligned}
$$

Finalmente, temos uma expressão para a volatilidade local da carteira dado um índice $B$, no instante inicial dada por:

$$
\sigma_{l o c}^{2}(0, B)=\sum_{i, j=1}^{n} \rho_{i j} \sigma_{i}\left(0, x_{i}^{*}\right) \sigma_{j}\left(0, x_{j}^{*}\right) p_{i}\left(0, \mathrm{x}^{*}\right) p_{j}\left(0, \mathrm{x}^{*}\right),
$$

em que $\lambda$ e $\mathrm{x}^{*}$ estão determinados pelas equações (2.38) e (2.39).

No seguinte capítulo estudamos as volatilidades implícitas dos ativos e a relação com a volatilidade implícita da carteira. Esta última relação é obtida passando pela expressão de $\sigma_{l o c}$. Uma vez resolvida tal conexão estaremos em condições de estudar dados de mercado. 


\section{Capítulo 3}

\section{Volatilidade implícita}

Estamos interessados em obter uma relação assintótica entre a volatilidade local e a volatilidade implícita, pois, por meio desta relação, podemos, a partir de dados do mercado e da fórmula (2.40), obter $\sigma_{l o c}$. Logo, tendo a volatilidade local da carteira e empregando novamente dita relação, calculamos a volatilidade implícita da carteira.

A complexidade de obter a relação mencionada se encontra no cálculo para $t=0$. Baseamosnos em um resultado recente que descreve assintóticamente a volatilidade implícita como a inversa da média harmônica da volatilidade local, corrigindo os problemas que apresentam outros métodos para $t \ll 1$.

\subsection{Fórmula de Dupire}

Antes de obter a mencionada relação, apresentamos de maneira sinóptica, como surgiu a idéia de pensar numa volatilidade local; já que permite modelar melhor que supondo volatilidade constante e, além disso, é mais fátivel de calcular que a volatilidade estocástica.

Em 1978, Breeden e Litzenberger provaram que a medida de Arrow Debreu ( probabilidade neutra ao risco) pode ser derivada dos retornos de 
opções européias. Em 1994, Dupire, Derman e Kani notaram que existe um único processo difusivo consistente com a medida de Arrow-Debreu. Assim, a função volatilidade local começou a ser interpretada como o único coeficiente de difusão dependente do estado e consistente com a aprecificação de opções europeas. Intuitivamente, estamos entendendo a volatilidade local como uma média sobre todas as possíveis volatilidades instantâneas em um "mundo estocástico". Desta maneira, a volatilidade local representa um modelo no qual o coeficiente de difusão dos retornos é dinâmico.

O trabalho que sintetiza o introduzido anteriormente, está dado pelo seguinte teorema.

Teorema 3.1.1 Se um mercado de opções sobre um ativo base com dinâmica de preços do tipo:

$$
\frac{d S_{\tau}}{S_{\tau}}=r d \tau+\sigma\left(\tau, S_{\tau}\right) d \mathbb{W}_{\tau}, \sigma: \mathbb{R} \times \mathbb{R} \rightarrow \mathbb{R}
$$

possui liquidez para todo vencimento $T$ e todo preço de exercício $K$, então existe uma única estrutura de volatilidade local compativel com o conjunto de preços $\{C(T, K)\}$ de opções negociados no mercado. Mais ainda, esta estrutura de volatilidade pode ser calculada explicitamente a partir dos preços das opções segundo a fórmula:

$$
\sigma(T, K)=\sqrt{\frac{C_{T}+r K C_{K}}{\frac{1}{2} K^{2} C_{K K}}}
$$

Demonstração.

O preço da opção pode ser escrito como o valor esperado sobre os possíveis preços do ativo, em que a densidade de probabilidade está dada pela medida de Arrow-Debreu $p(\tau, S, T, x)$

$$
\begin{aligned}
C(\tau, T, K) & =e^{-r(T-\tau)} \int_{-\infty}^{\infty}(x-K)_{+} p(\tau, S, T, x) d x \\
& =e^{-r(T-\tau)} \int_{K}^{\infty}(x-K) p(\tau, S, T, x) d x
\end{aligned}
$$

sendo $(x-K)_{+}=\max \{0, x-K\}$. 
Como estamos supondo um mercado líquido de opções em qualquer maturidade, podemos derivar a expressão anterior com relação à $T$

$$
\begin{aligned}
\frac{\partial C}{\partial T} & =-r e^{-r(T-\tau)} \int_{K}^{\infty}(x-K) p(\tau, S, T, x) d x \\
& +e^{-r(T-\tau)} \frac{\partial}{\partial T} \int_{K}^{\infty}(x-K) p(\tau, S, T, x) d x \\
& =-r C(\tau, T, K)+e^{-r(T-\tau)} \int_{K}^{\infty}(x-K) \frac{\partial p(\tau, S, T, x)}{\partial T} d x .
\end{aligned}
$$

Antes de continuar observemos que pelo resultado de Breeden e Litzenberger temos, dentro das hipóteses do Teorema de $\{C(T, K)\}$ contínuo, que a medida de Arrow-Debreu está dada por

$$
p(\tau, S, T, K)=e^{r(T-\tau)} \frac{\partial^{2} C}{\partial K^{2}} .
$$

Utilizando a equação de Kolmogorov direta (ver $\S 8$ [Øks00]),

$$
\frac{\partial p}{\partial T}=\frac{1}{2} \partial_{x x}^{2}\left(x^{2} \sigma^{2}(T, x) p\right)-\partial_{x}(r x p),
$$

a integral (3.3) resulta ser

$$
\begin{aligned}
C(\tau, T, K) & =e^{-r(T-\tau)}\left[\int_{K}^{\infty}(x-K) \partial_{x x}^{2}\left(x^{2} \sigma^{2}(T, x) p(\tau, S, T, x)\right) d x\right. \\
& \left.-\int_{K}^{\infty}(x-K) \partial_{x}(r x p(\tau, S, T, x)) d x\right]
\end{aligned}
$$

Aplicando integral por partes no primeiro termo

$$
\begin{aligned}
\int_{K}^{\infty}(x-K) & \partial_{x x}^{2}\left(x^{2} \sigma^{2}(T, x) p(\tau, S, T, x)\right) d x= \\
= & -\int_{K}^{\infty} \partial_{x}(x-K) \partial_{x}\left(x^{2} \sigma^{2}(T, x) p(\tau, S, T, x)\right) d x \\
& +\left.(x-K) \partial_{x}\left(x^{2} \sigma^{2}(T, x) p(\tau, S, T, x)\right)\right|_{K} ^{\infty} \\
= & -\int_{K}^{\infty} \partial_{x}\left(x^{2} \sigma^{2}(T, x) p(\tau, S, T, x)\right) d x \\
= & -\left.x^{2} \sigma^{2}(T, x) p(\tau, S, T, x)\right|_{K} ^{\infty} \\
= & K^{2} \sigma^{2}(T, K) p(\tau, S, T, K)
\end{aligned}
$$


e no segundo termo temos

$$
\begin{aligned}
-\int_{K}^{\infty}(x-K) \partial_{x}(\operatorname{rxp}(\tau, S, T, x)) d x & =\int_{K}^{\infty} \partial_{x}(x-K) \operatorname{rxp}(\tau, S, T, x) d x \\
& -\left.\operatorname{rxp}(\tau, S, T, x)(x-K)\right|_{K} ^{\infty} \\
& =r \int_{K}^{\infty} x p(\tau, S, T, x) d x
\end{aligned}
$$

Voltando para a derivada em $T$, resulta ser

$$
\begin{aligned}
\frac{\partial C}{\partial T} & =-r C+\frac{1}{2} K^{2} \sigma^{2}(T, K) \frac{\partial^{2} C}{\partial K^{2}}+r C+r K e^{-r(T-\tau)} \int_{K}^{\infty} p(\tau, S, T, x) d x \\
& =\frac{1}{2} K^{2} \sigma^{2}(T, K) \frac{\partial^{2} C}{\partial K^{2}}+r K \frac{\partial C}{\partial K} .
\end{aligned}
$$

Isolando o termo da volatilidade, obtemos a fórmula de Dupire dada por:

$$
\sigma^{2}(T, K)=\frac{C_{T}+r K C_{K}}{\frac{1}{2} K^{2} C_{K K}}
$$

A expressão (3.1) pode ser vista como "definição" da volatilidade local, independendo do tipo de processo que verdadeiramente determina a estrutura de volatilidade.

A importância da fórmula de Dupire subjace em determinar de maneira única e explícita a volatilidade local. Deste modo, é possível obter uma relação ( fórmula fechada) entre a volatilidade local e a volatilidade implícita (ver $\S 2.3$ [Gat01]). No entanto, esse desenvolvimento é em um contexto de um "mundo teórico" onde as opções europeas são apreçadas para todos os possíveis valores de exercício e todas as maturidades. Na realidade não é assim, pois $\{C(T, K)\}$ é discreto ${ }^{1}$. Podem ser utilizadas ferramentas de interpolação, embora não é obvia a escolha do método para interpolar os dados fornecidos pelo mercado, para opções de curta expiração, isto é quando $t=T-\tau \ll 1$. Neste caso, os resultados são muito sensíveis à escolha do método de interpolação. Outra dificuldade é que nas regiões

\footnotetext{
${ }^{1}$ Uma versão discreta do trabalho de Dupire é dada pelas árvores de Derman-Kani
} 
$\{T-\tau \ll 1\},\left\{\left|\ln \left(S_{0} / K\right)\right| \gg 1\right\},\{T-\tau \gg 1\}$ a fórmula de Dupire apresenta indeterminações do tipo $\frac{0}{0}$.

Para conseguir a relação desejada entre a volatilidade implícita e a local, evitando supor $\{C(T, K)\}$ contínuo, apresentamos, na seguinte seção, um novo ponto de vista do problema. É um resultado que mostra a existência de uma conexão explícita entre as duas volatilidades, tendo esta a forma da solução de uma EDP parabólica degenerada de tipo quase-linear.

\subsection{Relação assintótica entre volatilidade local e implícita}

Nesta seção procuramos uma função que relacione a volatilidade local com a volatilidade implícita. Vamos mostrar o surpreendente resultado que afirma ser a volatilidade implícita uma espécie de "média harmônica" da volatilidade local, no limite quando $t \downarrow 0$, isto é:

$$
\sigma_{I}\left(0^{+}, x\right)=\lim _{t \rightarrow 0^{+}} \sigma_{I}(t, x)=\left(\int_{0}^{1} \frac{d s}{\sigma_{l o c}(0, s x)}\right)^{-1} .
$$

Os cálculos serão feitos formalmente, ignorando aspectos ligados à regularidade das soluções de certas EDPs. Um desenvolvimento rigoroso pode ser encontrado em [BBF02].

No modelo de Black-Scholes o preço $C=C(\tau, S ; T, K)$ de um Call europeu com o valor do ativo $S_{\tau}$ está dado pela solução da seguinte EDP:

$$
\left\{\begin{array}{l}
C_{\tau}+\frac{\sigma^{2}}{2} S^{2} C_{S S}+r S C_{S}-r C=0 \quad(0, T) \times(0,+\infty), \\
C(T, S)=(S-K)_{+},
\end{array}\right.
$$

onde $T$ é a data de vencimento do título, $r$ a taxa livre de risco e $K$ o valor de exercício. A volatilidade local está denotada por $\sigma$.

Aplicando a mudança de variáveis $x=\ln (S / K)+r t, t=T-\tau$ o valor do call resulta ser $\left.v(t, x)=e^{r t} C\left(e^{x-r t} K, T-t ; K, T\right) / K\right)$ em que $v$ satisfaz, 
a transformação da equação (3.4) dada por:

$$
\left\{\begin{array}{l}
v_{t}=\frac{1}{2} \sigma^{2}(t, x)\left(v_{x x}-v_{x}\right) \quad \text { em } \Omega_{T}=(0, T) \times \mathbb{R} \\
v(0, x)=\left(e^{x}-1\right)_{+}
\end{array}\right.
$$

Denotamos por $u$ a solução da $\operatorname{EDP}$ (3.5) para o caso $\sigma^{2} \equiv 1$. A partir de $u$ e $v$ definiremos a volatilidade implícita com um esquema semelhante ao método de variação de parâmetros para equações diferenciais.

Observemos que a aplicação $v(t, x)=u(\lambda t, x)$ satisfaz a EDP (3.5) para $\sigma \equiv \sqrt{\lambda}$. Isto nos sugere definir uma $\phi \geq 0$ tal que

$$
v(t, x)=u\left(t \phi^{2}(t, x), x\right)
$$

é solução de (3.5), e dita $\phi$ é a volatilidade implícita procurada. Denotamos $\phi(t, x)$ por $\sigma_{I}(t, x)$.

Da definição da $\sigma_{I}$ e da $\operatorname{EDP}(3.5)$ obtemos

$$
\begin{aligned}
v_{t}-\frac{1}{2} \sigma^{2}(t, x)\left(v_{x x}-v_{x}\right) & =u_{t} F\left(t, x, \sigma_{I}, D \sigma_{I}, D^{2} \sigma_{I}\right) \\
0 & \equiv u_{t} F\left(t, x, \sigma_{I}, D \sigma_{I}, D^{2} \sigma_{I}\right)
\end{aligned}
$$

na qual

$$
\begin{aligned}
F\left(t, x, \sigma_{I}, D \sigma_{I}, D^{2} \sigma_{I}\right)= & -\sigma^{2} t \sigma_{I} \partial_{x x}^{2} \sigma_{I}+\frac{\sigma^{2}}{4} t^{2} \sigma_{I}^{2}\left(\partial_{x} \sigma_{I}\right)^{2} \\
& -\sigma^{2}\left(1-x \frac{\partial_{x} \sigma_{I}}{\sigma_{I}}\right)^{2}+2 t \sigma_{I} \partial_{t} \sigma_{I}+\sigma_{I}^{2}
\end{aligned}
$$

Lembramos que $u$ foi definida como a solução de (3.5) para $\sigma \equiv 1$, isto é, $u(t, x)$ é solução de

$$
\left\{\begin{array}{l}
u_{t}=\frac{1}{2}\left(u_{x x}-u_{x}\right) \quad \text { em } \Omega_{T} \\
u(0, x)=\left(e^{x}-1\right)_{+}
\end{array}\right.
$$

satisfazendo $u_{t}>0$. Logo, de (3.7) deduzimos que $\sigma_{I}$ está dada pela solução de $F \equiv 0$. Dentro do contexto mais rigoroso de [BBF02] pode-se provar que 
$\sigma_{I}$ é a solução de um problema parabólico quase-linear degenerado definido pelo operador $F$.

Como procuramos obter $\sigma_{I}$ em função de $\sigma$, pomos em evidência $\sigma$ na equação $F \equiv 0$ e obtemos a seguinte aplicação

$$
\sigma[\psi](t, x)=\sqrt{\frac{\partial_{t}\left(t \sigma_{I}^{2}\right)}{H\left(t, x, \psi, D \psi, D^{2} \psi\right)}}
$$

na qual,

$$
H\left(t, x, \sigma_{I}, D \psi, D^{2} \psi\right)=t \psi \partial_{x x}^{2} \psi-\frac{1}{4} t^{2} \psi^{2}\left(\partial_{x} \psi\right)^{2}+\left(1-x \frac{\partial_{x} \psi}{\psi}\right)^{2} .
$$

Ou seja, $\sigma_{I}$ é solução da equação $\sigma[\psi] \equiv \sigma$.

Podemos definir formalmente a solução limite $\phi^{0}$ do operador $F \equiv 0$, ou seja,

$$
\left(\phi^{0}\right)^{2}-\sigma^{2}(0, x)\left(1-x \frac{\phi_{x}^{0}}{\phi^{0}}\right)^{2}=0
$$

cuja forma explícita é:

$$
\phi^{0}(x)=\left(\int_{0}^{1} \frac{d s}{\sigma(0, s x)}\right)^{-1}
$$

A expressão anterior é obtida aplicando a mudança $\phi^{0}=x \xi(x)$ e logo o método de separação de variáveis.

Definimos funções inferior e superior à nossa $\sigma_{I}$ e utilizando um princípio de comparação, obtemos a expressão para a volatilidade implícita. Antes de continuar estudemos tal princípio.

Lema 3.2.1 (Princípio de Dominação) Se $\psi_{1}$ e $\psi_{2} \in \mathcal{C}^{2}\left(\Omega_{T}\right) \cap \mathcal{C}\left(\bar{\Omega}_{T}\right)$, verificam a relação de dominação de volatilidade local, isto é:

$$
\sigma\left[\psi_{1}\right](t, x) \leq \sigma\left[\psi_{2}\right](t, x) \quad \forall(t, x) \in \Omega_{T},
$$

então $\psi_{1} \leq \psi_{2}, \forall(t, x) \in \Omega_{T}$. 


\section{Demonstração.}

Definimos $\underline{u}(t, x)=u\left(t \psi_{1}^{2}(t, x), x\right)$ e $\bar{u}(t, x)=u\left(t \psi_{2}^{2}(t, x), x\right)$ satisfazendo, respectivamente,

$$
\left\{\begin{array}{l}
\underline{u}_{t} \frac{1}{2} \sigma\left[\psi_{1}\right]^{2}(t, x)\left(\underline{u}_{x x}-\underline{u}_{x}\right)=0 \quad \text { em } \Omega_{T} \\
\underline{u}(0, x)=\left(e^{x}-1\right)_{+}
\end{array}\right.
$$

e

$$
\left\{\begin{array}{l}
\bar{u}_{t}-\frac{1}{2} \sigma\left[\psi_{2}\right]^{2}(t, x)\left(\bar{u}_{x x}-\bar{u}_{x}\right)=0 \quad \text { em } \Omega_{T} \\
\bar{u}(0, x)=\left(e^{x}-1\right)_{+}
\end{array}\right.
$$

Para estabelecer uma comparação, definimos a função diferença $w(t, x)=$ $\bar{u}(t, x)-\underline{u}(t, x)$, a qual satisfaz

$$
\begin{aligned}
w_{t}=\bar{u}_{t}-\underline{u}_{t}= & \frac{1}{2}\left(\sigma\left[\psi_{2}\right]^{2}(t, x) \cdot\left(\bar{u}_{x x}-\bar{u}_{x}\right)-\sigma\left[\psi_{1}\right]^{2}(t, x) \cdot\left(\underline{u}_{x x}-\underline{u}_{x}\right)\right) \\
= & \frac{1}{2}\left[\sigma\left[\psi_{2}\right]^{2}(t, x) \cdot\left(w_{x x}-w_{x}\right)+\sigma\left[\psi_{2}\right]^{2}(t, x) \cdot\left(\underline{u}_{x x}-\underline{u}_{x}\right)\right. \\
& -\sigma\left[\psi_{1}\right]^{2}(t, x) \cdot\left(\underline{u}_{x x}-\underline{u}_{x}\right) .
\end{aligned}
$$

Ou seja, $w$ é solução de

$$
\left\{\begin{array}{l}
w_{t}-\frac{1}{2} \sigma\left[\psi_{2}\right]^{2}(t, x) \cdot\left(w_{x x}-w_{x}\right)=\underline{u}_{t} \cdot\left(\frac{\sigma\left[\psi_{2}\right]^{2}}{\sigma\left[\psi_{1}\right]^{2}}-1\right) \text { em } \Omega_{T} \\
w(0, x)=0 .
\end{array}\right.
$$

Por (3.9) e pela definição de $u$ deduzimos que o lado direito é positivo e, portanto, o esquerdo também; logo, podemos aplicar o Princípio do Máximo para equações parabólicas e concluir que $w \geq 0 \mathrm{em} \Omega_{T}$. Por outro lado, como $u$ é uma função crescente na variável $t\left(u_{t}>0\right)$ concluímos que $\psi_{1} \leq \psi_{2}$ em $\Omega_{T}$.

Dado $\kappa>0$ definimos

$$
\begin{aligned}
& \bar{\phi}(t, x)=\phi^{0}(x)(1+\kappa t), \\
& \underline{\phi}(t, x)=\phi^{0}(x)(1-\kappa t) .
\end{aligned}
$$


Queremos provar que

$$
\bar{\phi}(t, x) \leq \sigma_{I}(t, x) \leq \bar{\phi}(t, x),
$$

para logo aplicar limite de $t \downarrow 0$ e obter a expressão assintótica para $\sigma_{I}$.

Provando que $\sigma[\phi] \leq \sigma\left[\sigma_{I}\right] \leq \sigma[\bar{\phi}]$, e aplicando o lema 3.2.1, obtemos a desigualdade (3.10), pois $\sigma\left[\sigma_{I}\right] \equiv \sigma$.

Calculemos $\sigma[\bar{\phi}]$, o operador $H$ fica:

$$
\begin{aligned}
H\left(t, x, \bar{\phi}, D \bar{\phi}, D^{2} \bar{\phi}\right) & =t \bar{\phi} \partial_{x x}^{2} \bar{\phi}-\frac{1}{4} t^{2} \bar{\phi}^{2}\left(\partial_{x} \bar{\phi}\right)^{2}+\left(1-x \frac{\partial_{x} \bar{\phi}}{\bar{\phi}}\right)^{2} \\
& =t \phi^{0} \partial_{x x}^{2} \phi^{0}(1+\kappa t)^{2}-\frac{1}{4}\left(t \phi^{0} \partial_{x} \phi^{0}\right)^{2}(1+\kappa t)^{4} \\
& +\left(1-x \frac{\partial_{x} \phi^{0}}{\phi^{0}}\right)^{2} \\
& =t \phi^{0} \partial_{x x}^{2} \phi^{0}+\left(1-x \frac{\partial_{x} \phi^{0}}{\phi^{0}}\right)^{2} \\
& +t^{2}\left(2 \kappa \phi^{0} \partial_{x x}^{2} \phi^{0}+\kappa t \phi^{0} \partial_{x x}^{2} \phi^{0}-\frac{1}{4}\left(\phi^{0} \partial_{x} \phi^{0}\right)^{2}(1+\kappa t)^{4}\right) \\
& =t \phi^{0} \partial_{x x}^{2} \phi^{0}+\left(1-x \frac{\partial_{x} \phi^{0}}{\phi^{0}}\right)^{2}+O\left(t^{2}\right) .
\end{aligned}
$$

Semelhantemente, para a derivada em relação à $t$, temos:

$$
\begin{aligned}
\partial_{t}\left(t \bar{\phi}^{2}\right) & =(1+\kappa t)^{2}\left(\phi^{0}\right)^{2} \\
& =\phi^{0^{2}}(1+\kappa t)(1+3 \kappa t) .
\end{aligned}
$$

Por outro lado,

$$
\sigma^{2}(t, x)=\sigma^{2}(0, x)+2 \sigma(0, x) \sigma_{t}(0, x) t+O\left(t^{2}\right) .
$$

Portanto,

$$
\begin{aligned}
F\left(t, x, \bar{\phi}, D \bar{\phi}, D^{2} \bar{\phi}\right) & =\underbrace{\partial_{t}\left(t \bar{\phi}^{2}\right)-\sigma^{2}(t, x) H\left(t, x, \bar{\phi}, D \bar{\phi}, D^{2} \bar{\phi}\right)}_{=0} \\
& =\underbrace{0^{2}-\sigma^{2}(0, x)\left(1-x \frac{\partial_{x} \phi^{0}}{\phi^{0}}\right)^{2}} \\
& +t \phi^{0^{2}}\left(4 \kappa-\sigma^{2}(t, x) \frac{\partial_{x x}^{2} \phi^{0}}{\phi^{0}}-2 \frac{\sigma_{t}}{\sigma}(0, x)\right)+O\left(t^{2}\right) .
\end{aligned}
$$


Da equação anterior, para $\kappa$ suficientemente grande e $t \ll 1$, deduzimos que $F\left(t, x, \bar{\phi}, D \bar{\phi}, D^{2} \bar{\phi}\right) \geq 0$, ou seja,

$$
\partial_{t}\left(t \bar{\phi}^{2}\right) \geq \sigma^{2}(t, x) H\left(t, x, \bar{\phi}, D \bar{\phi}, D^{2} \bar{\phi}\right)
$$

por tanto:

$$
\sigma[\bar{\phi}] \geq \sigma(t, x) .
$$

Analogamente, podemos provar que $\sigma[\underline{\phi}] \leq \sigma(t, x)$, concluindo que:

$$
\underline{\phi}(t, x) \leq \sigma_{I}(t, x) \leq \bar{\phi}(t, x) .
$$

Pela definição de $\bar{\phi}$ e $\underline{\phi}$ obtemos:

$$
\phi^{0}(x)(1-\kappa t) \leq \sigma_{I}(t, x) \leq \phi^{0}(x)(1+\kappa t) .
$$

Tomando o limite quando $t \downarrow 0$

$$
\phi^{0}(x) \leq \lim _{t \rightarrow 0} \sigma_{I}(t, x) \leq \phi^{0}(x),
$$

e portanto

$$
\sigma_{I}\left(0^{+}, x\right)=\phi^{0}(x)
$$

Finalmente, obtemos a expressão para a volatilidade implícita no limite para $t \downarrow 0$

$$
\sigma_{I}\left(0^{+}, x\right)=\left(\int_{0}^{1} \frac{d s}{\sigma(0, s x)}\right)^{-1}
$$

\subsection{Volatilidade implícita da carteira}

Nesta seção aplicamos à carteira a relação obtida entre a volatilidade local e a implícita. Para isto, escrevemos a integral (3.13) como:

$$
\sigma_{I}\left(0^{+}, x\right)=\left(\frac{1}{x} \int_{0}^{x} \frac{d u}{\sigma(0, u)}\right)^{-1} .
$$

A seguir provamos que podemos aproximar a integral anterior por uma média aritmética, obtendo assim uma fórmula simples de trabalhar. 
Para $|x| \rightarrow 0$ a integral em (3.14) pode ser aproximada pela expressão:

$$
\frac{1}{x} \int_{0}^{x} \frac{d u}{\sigma(0, u)}=\frac{1}{2}\left(\frac{1}{\sigma(0, x)}+\frac{1}{\sigma(0,0)}\right)+O(|x|) .
$$

Por outro lado, como $\sigma(0, x)=\sigma(0,0)+O(|x|)$, a inversa da média harmônica é próxima à média aritmética, pois, denotando $O(|x|)$ por $\epsilon$, temos:

$$
\begin{aligned}
2\left(\frac{1}{\sigma(0,0)+\epsilon}+\frac{1}{\sigma(0,0)}\right)^{-1} & -\left(\frac{(\sigma(0,0)+\epsilon)+\sigma(0,0)}{2}\right)= \\
& =\frac{2 \sigma(0,0)(\sigma(0,0)+\epsilon)}{2 \sigma(0,0)+\epsilon}-\frac{2 \sigma(0,0)+\epsilon}{2} \\
& =-\frac{\epsilon^{2}}{4 \sigma(0,0)+2 \epsilon} \\
& =o(\epsilon) .
\end{aligned}
$$

obtendo a fórmula aproximada:

$$
\sigma_{I}(0, x)=\frac{\sigma(0, x)+\sigma(0,0)}{2} .
$$

Portanto, para cada ativo obtemos as aproximações:

$$
\sigma_{i}\left(0, x_{i}^{*}\right) \approx 2 \sigma_{i}^{I}\left(0, x_{i}^{*}\right)-\sigma_{i}^{I}(0,0) \quad i=1, \ldots, n .
$$

Substituindo na equação (2.40), obtemos a seguinte equação para a volatilidade local do índice:

$$
\begin{aligned}
& \sigma_{l o c}(0, B) \approx \\
& \quad \sqrt{\sum_{i, j=1} \rho_{i j} p_{i}\left(0, \mathrm{x}^{*}\right) p_{j}\left(0, \mathrm{x}^{*}\right)\left(2 \sigma_{i}^{I}\left(0, x_{i}^{*}\right)-\sigma_{i}^{I}(0,0)\right)\left(2 \sigma_{j}^{I}\left(0, x_{j}^{*}\right)-\sigma_{j}^{I}(0,0)\right)} .
\end{aligned}
$$

Finalmente, podemos escrever a volatilidade implícita da carteira para um índice $B$ :

$$
\sigma_{I}(B) \approx \frac{\sigma_{l o c}(0, B)+\sigma_{0}(0)}{2}
$$

Observamos que por definição $\sigma_{l o c}(0,0)=\sigma_{0}(0)$. 


\section{Capítulo 4}

\section{Aplicação para o mercado brasileiro de derivativos}

No presente capítulo construímos uma carteira formada pelas ações cujas opções foram as mais líquidas no período de 3 de junho até o 4 de julho, a saber, Petrobrás e Telesp. Também calculamos smiles em fução das diferentes participações das duas ações. Por isto, é necessário fazer uma análise das estimativas do coeficiente de correlação entre as duas ações.

\subsection{Estimação da correlação entre os ativos}

\subsubsection{Estimativa intra-diária}

Os dados que empregamos são do mês de junho de 2002 e são cotados a cada vinte minutos. Observamos que todos os dados foram coletados em tempo real de mercado, garantindo seu sincronismo.

Empregamos as ações da Petrobrás (PETR4) e Telesp participações (TSPP4) para realizar nossos exemplos devido à maior liquidez de suas opções. O primeiro passo será determinar o coeficiente de correlação $\rho$, entre os retornos destas ações. Quando calculamos a correlação intradiária, isto é, a correlação dos retornos intradiários dos ativos estimada ao final do 
dia, observamos que o coeficiente de correlação muda razoavelmente entre os dias analisados (figura 4.1). Em alguns dias as ações apresentam correlação próxima da unidade e em outros dias, apresentam correlação negativa. Estes últimos valores são duvidosos já que, em geral, espera-se correlação positiva, pois todo o mercado está em alta ou todo está em baixa. Interpretamos que nesses dias algum fato idiossincrático influiu negativamente em só uma das ações. Assim, deduzimos que a estimação intradiária pode ser fina demais.

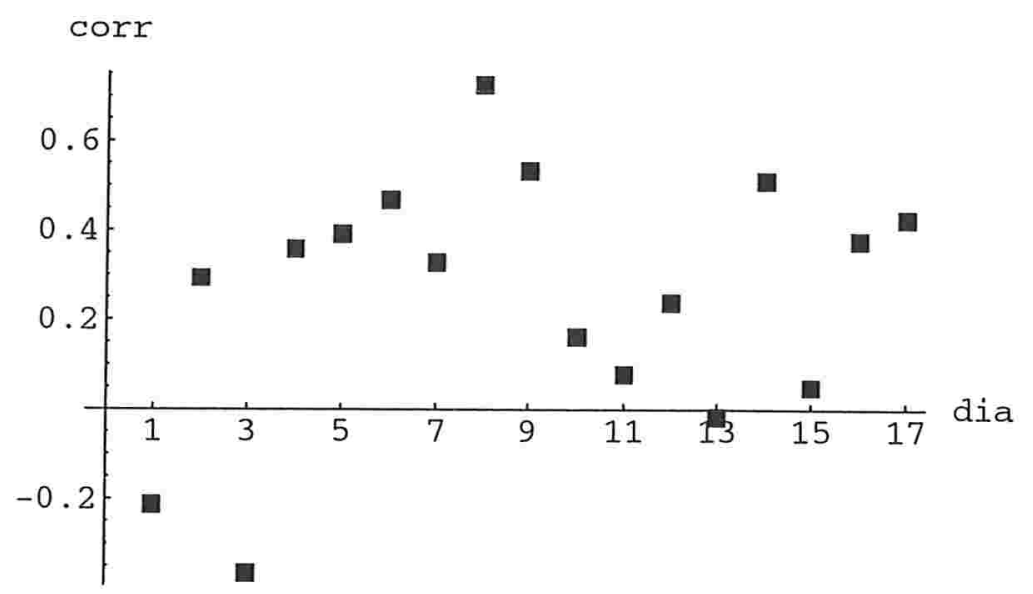

Figura 4.1

Correlações intradiárias entre PETR4 e TSPP4 para 17 dias observados.

\subsubsection{Estimativa diária}

Como segunda forma de cálculo podemos estimar a cotação média do dia e calcular a correlação entre as médias diárias, obtendo um único valor global:

$$
\rho=0,3368 .
$$

Uma interpretação das diferenças nos valores das correlações intradiárias e as diárias é que, no caso das intradiárias estamos olhando microscopica- 
mente, percebendo efeitos que no caso de correlações diárias são praticamente ignorados. Por exemplo, algumas notícias demoram dias para serem absorvidas pelo mercado e por isto, o cálculo de $\rho$ naqueles dias pode mudar ınuito em relação à dias anteriores ou posteriores.

Em virtude de termos obtido valores muito diferentes dependendo da freqüência de observação, surge a questão de estudar o impacto do coeficiente de correlação na volatilidade implícita da carteira. Com tal fim definimos uma carteira qualquer, com participação fixadas, e calculamos os smiles de volatilidade da carteira para diferentes valores de $\rho$.

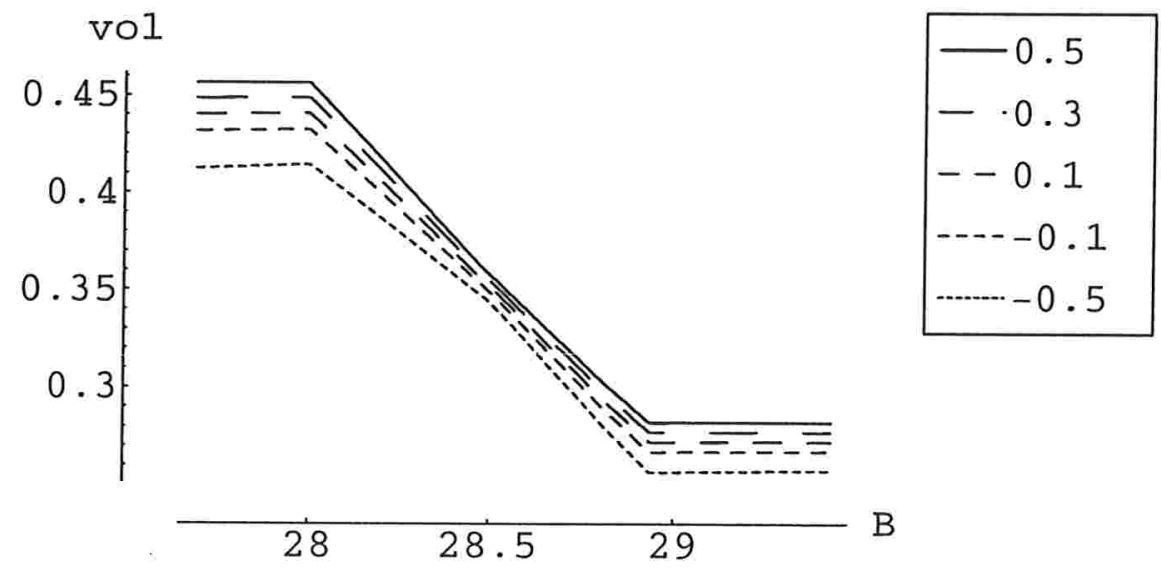

Figura 4.2

Sensibilidade do smile de uma carteira com relação ao coeficiente de correlação entre as ações que a definem.

Na figura 4.2 podemos observar que o cálculo do smile de um índice é sensível ao valor da correlação. Podemos concluir que é importante fazer uma correta estimação da correlação, uma vez que sua influência no cálculo da volatilidade do índice é grande.

Como conclusão, sugerimos que a determinação dos coeficientes de correlação seja feita através de uma precissa análise econométrica dos dados. Esta análise, tão complexa quanto interessante, foge ao escorpo do trabalho e 
para não desviarmos do objetivo central, deixamos a questão das correlações como tema para uma futura pesquisa, e empregando a correlação de toda a amostra. Isto é feito, justamente, por não nos importarmos com as questões econométricas. Assim, os exemplos da seçãc seguinte serão calculados com

$$
\operatorname{Corr}(\text { PETR4, TSPP4) }=0,2924 .
$$

Havendo estabelecido o coeficiente de correlação calculamos, na seguinte seção, a volatilidade smile das carteiras.

\subsection{Smiles de volatilidade}

Nesta seção calculamos o smile de uma carteira de ações de PETR4 e de TSPP4, com diferentes participações.

Antes de apresentar os resultados obtidos, definimos alguns critérios para os cálculos. Quando fazemos referência ao smile, estamos falando da curva obtida por meio de uma interpolação linear das volatilidades implícitas fornecidas. Por outro lado, em nosso conjunto de dados, só temos uma data de maturidade $T$ para cada opção. Portanto, para obter a curva smile de opções que vencem no momento do cálculo, $t=0$, simplesmente assumimos que a superfície de volatilidade do ativo não possui curvatura na direção do tempo, isto é, as volatilidades implícitas de opções com maturidade $T$ serão vistas também como as volatilidades no instante $t=0$.

Denotaremos por $A T M$ o valor do índice no dinheiro, isto é, quando $B=B(0,0)$.

As análises apresentadas a seguir foram realizados com dados do dia 4 de julho de 2002. Na tabela 4.1 e na figura 4.3 calculamos a volatilidade implícita ATM de carteiras com diferentes participações das ações. 
Tabela 4.1

Incidência das participações efetivas das ações na volatilidade implícita ATM de uma carteira. Para PETR4: $S(0)=46,78$ e $\sigma_{I}(A T M)=$ 0, 2441, TSPP4: $S(0)=3,73$ e $\sigma_{I}(A T M)=0,461$.

\begin{tabular}{|c|c|c|c|c|}
\hline \multicolumn{2}{|c|}{ PETR4 } & \multicolumn{2}{|c|}{ TSPP4 } & \multirow{2}{*}{$\sigma_{I}\left(B_{\text {ATM }}\right)$} \\
\cline { 1 - 4 }$w$ & $p(0, S(0))$ & $w$ & $p(0, S(0))$ & \\
\hline \hline $100 \%$ & 1 & $0 \%$ & 0 & 0,2441 \\
$90 \%$ & 0,991 & $10 \%$ & 0,009 & 0,2431 \\
$75 \%$ & 0,975 & $25 \%$ & 0,025 & 0,2415 \\
$50 \%$ & 0,929 & $50 \%$ & 0,071 & 0,2382 \\
$25 \%$ & 0,807 & $75 \%$ & 0,193 & 0,2387 \\
$10 \%$ & 0,582 & $90 \%$ & 0,418 & 0,2707 \\
$8 \%$ & 0,522 & $92 \%$ & 0,478 & 0,2850 \\
$6 \%$ & 0,445 & $94 \%$ & 0,555 & 0,3059 \\
$4 \%$ & 0,343 & $96 \%$ & 0,657 & 0,3369 \\
$2 \%$ & 0,204 & $98 \%$ & 0,796 & 0,3845 \\
$1 \%$ & 0,112 & $99 \%$ & 0,888 & 0,4180 \\
$0 \%$ & 0 & $100 \%$ & 1 & 0,4610 \\
\hline
\end{tabular}

Tanto na tabela 4.1 quanto na figura 4.3 podemos observar que a ação TSPP4 tem participação efetiva importante $\left(p_{T S P P 4}(0, S)>0.55\right)$ quando a carteira consiste predominantemente das ditas ações. Isto gera um salto pronunciado na volatilidade implícita. 

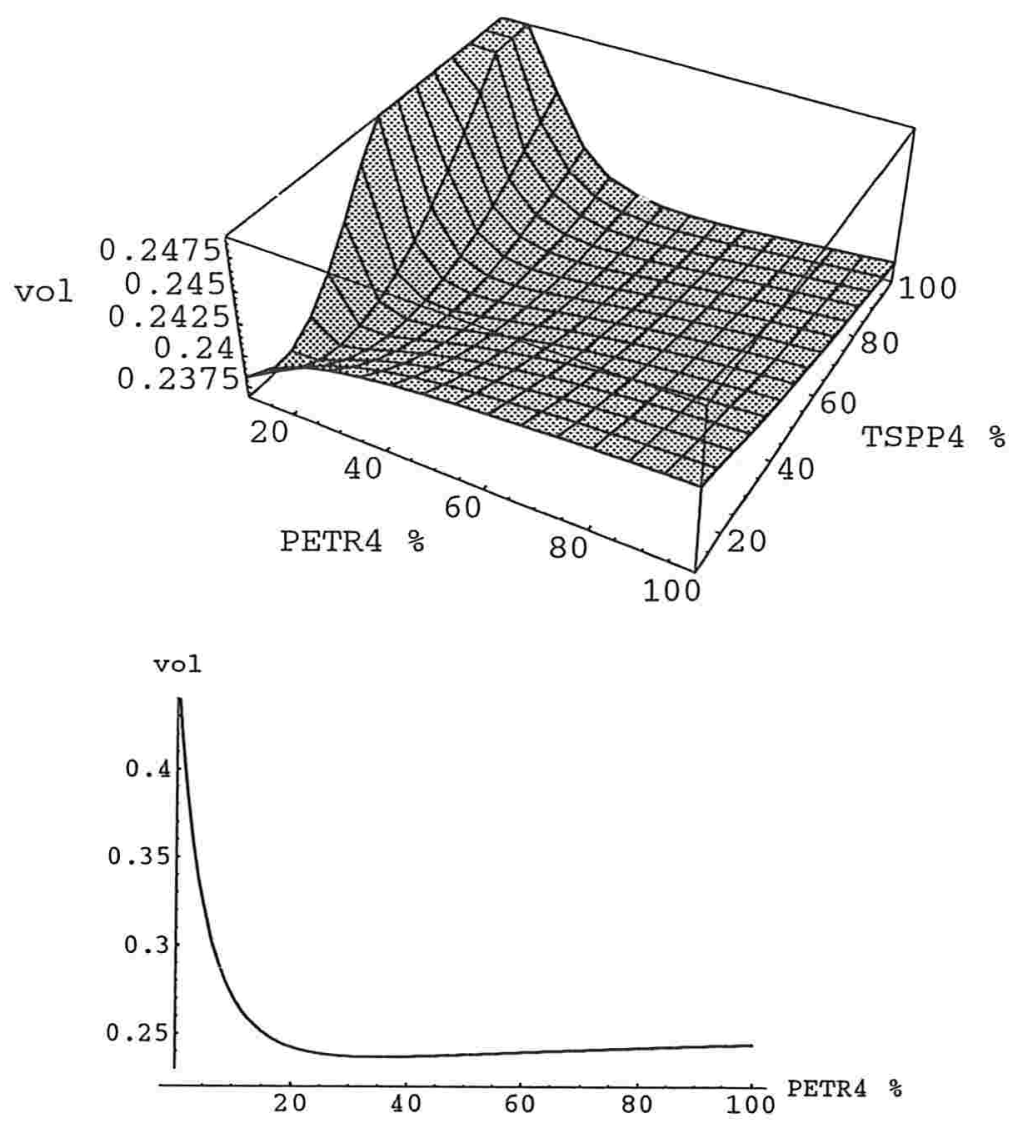

Figura 4.3

Volatilidade implícita de uma carteira como função das diferentes porcentagens das ações que a definem.

A seguir apresentamos três fotografias das volatilidades de dias diferentes. Em cada uma delas desenhamos as volatilidades das ações PETR4 e TSPP4 para as observações de mercado realizadas à cada vinte minutos. Também desenhamos a volatilidade de uma carteira definida por $50 \%$ das ações mencionadas. Tanto as volatilidades implícitas das ações quanto do índice são calculadas para valores no dinheiro (ATM). 

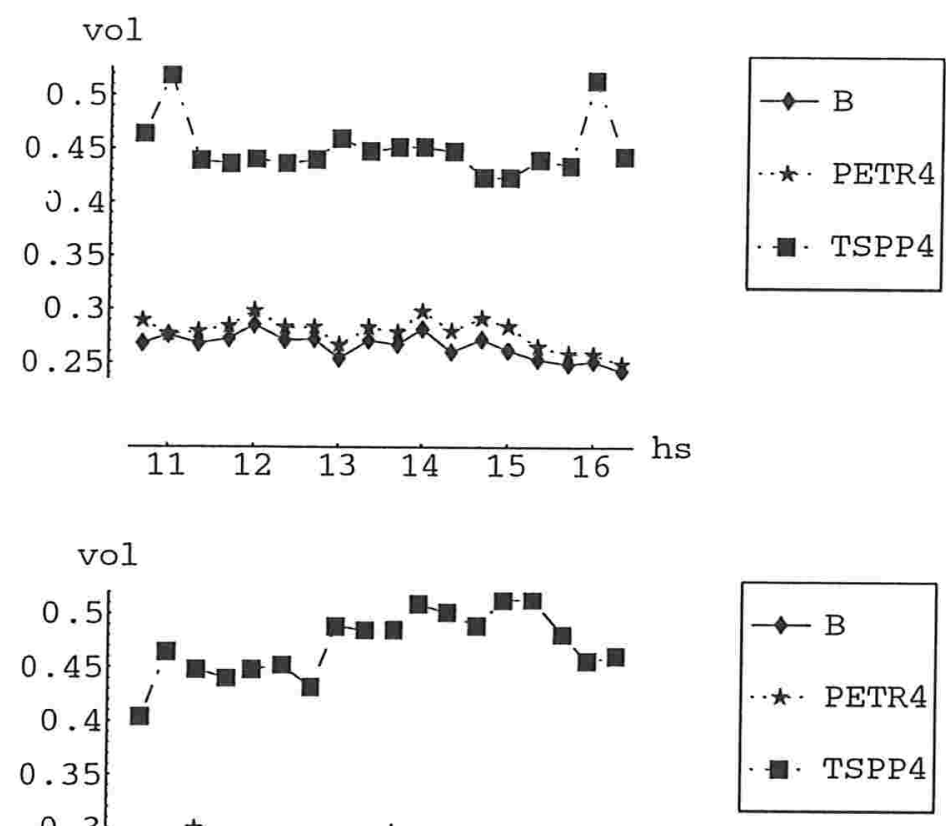

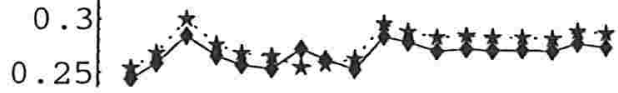
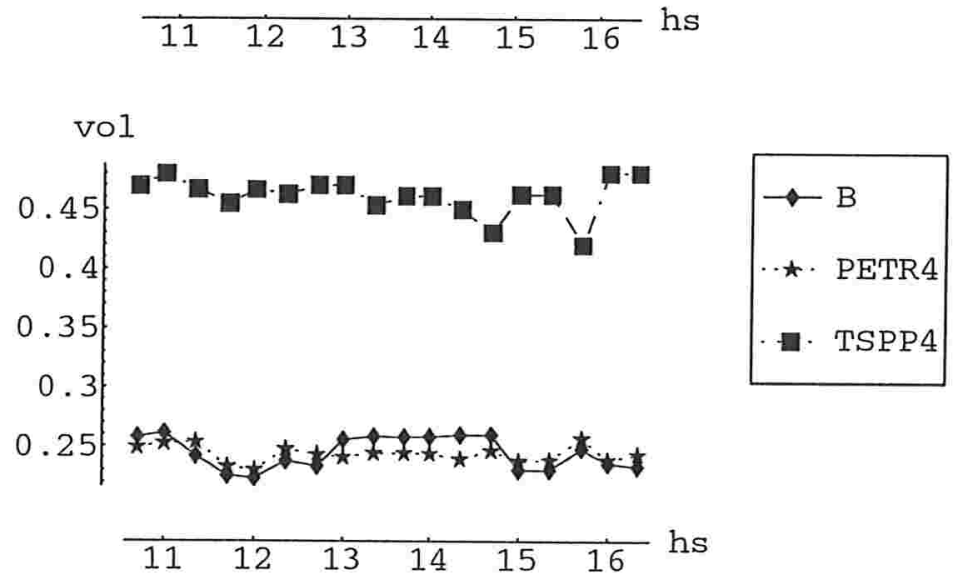

Figura 4.4

Curvas de volatilidade das ações PETR4, TSPP4 e da carteira definida com $50 \%$ de cada ação. Valores ATM para o dia 2, 3 e 4 de julho de 2002, com obserrvações cada 20 minutos. 
Na figura 4.4 observamos novamente que a volatilidade da carteira no dinheiro tem um comportamento semelhante àquele definido pela ação PETR4. O fato de ter tal ação maior incidência na volatilidade implícita do índice também pode ser observado na tabela 4.2.

Tabela 4.2

Volatilidades de índices de uma carteira de 50\% PETR4 e 50\% TSPP4, com observações realizadas no dia 4 de julho de 2002 às 14:00hs.

\begin{tabular}{|l|c|l|c|l|c|}
\hline \multicolumn{2}{|c|}{ TSPP4 } & \multicolumn{2}{c|}{ PETR4 } & \multicolumn{2}{c|}{ carteira } \\
\hline $\mathrm{K}$ & $\sigma_{I}$ & $\mathrm{~K}$ & $\sigma_{I}$ & $\mathrm{~B}$ & $\sigma_{I}$ \\
\hline \hline 4 & 0,4702 & 48 & 0,2437 & 26 & 0,2579 \\
4 & 0,4702 & 50 & 0,2466 & 27 & 0,2839 \\
4 & 0,4702 & 52 & 0,2617 & 28 & 0,2847 \\
4 & 0,4702 & 53,87 & 0,2761 & 28,93 & 0,2853 \\
4 & 0,4702 & 54 & 0,2768 & 29 & 0,2854 \\
4 & 0,4702 & 56 & 0,2889 & 30 & 0,2859 \\
4 & 0,4702 & 58 & 0,2939 & 31 & 0,2867 \\
5 & 0,5195 & 48 & 0,2437 & 26,5 & 0,2813 \\
5 & 0,5195 & 50 & 0,2466 & 27,5 & 0,2843 \\
5 & 0,5195 & 52 & 0,2617 & 28,5 & 0,2850 \\
5 & 0,5195 & 53,87 & 0,2761 & 29,43 & 0,2856 \\
5 & 0,5195 & 54 & 0,2768 & 29,5 & 0,2857 \\
5 & 0,5195 & 56 & 0,2889 & 30,5 & 0,2863 \\
5 & 0,5195 & 58 & 0,2939 & 31,5 & 0,2870 \\
6 & 0,6025 & 48 & 0,2437 & 27 & 0,2839 \\
6 & 0,6025 & 50 & 0,2466 & 28 & 0,2847 \\
6 & 0,6025 & 52 & 0,2617 & 29 & 0,2854 \\
6 & 0,6025 & 53,87 & 0,2761 & 29,93 & 0,2859 \\
6 & 0,6025 & 54 & 0,2768 & 30 & 0,2859 \\
6 & 0,6025 & 56 & 0,2889 & 31 & 0,2867 \\
6 & 0,6025 & 58 & 0,2939 & 32 & 0,2872 \\
\hline
\end{tabular}


A tabela 4.2 apresenta os valores das volatilidades implícitas dos preços de exercício das opções cotizadas no mercado sobre as ações estudadas. As convinações de tais grandezas definem os índices para os quais é interessante corrhecer a volatilidade implícita.

Para finalizar esta seção, gostaríamos de salientar que um estudo mais aprofundado desta técnica depende de observamos no mercado um número maior de opções líquidas. Com isto, poderiamos inferir melhor os papéis das correlações neste estudo, assim como obter melhores aplicações do método. 


\section{Conclusão}

Neste trabalho foi apresentado um método de reconstrução da estrutura de volatilidade de uma carteira de ativos de renda variável. Tal reconstrução foi realizada por meio de uma aproximação assintótica por volatilidades dos ativos em valores ótimos.

O método aqui estudado, está baseado no artigo [Ave02]. A contribuição da presente dissertação consiste em considerar os resultados expostos no artigo mencionado e fazer uma análise exaustiva, incluindo demonstrações e explicações dos resultados, assim como de aplicá-los ao mercado brasileiro de derivativos. Infelizmente, a falta de liquidez deste mercado impossibilita testes mais conclusivos sobre a eficácia do método.

Como consideração final, salienta-se que o presente trabalho permite analisar um parâmetro tão complexo quanto essencial como é a volatilidade. Tal análise precisa uma compreensão tanto da abordagem matemática quanto da realidade financeira subjacente, principalmente na fase de interpretação a sintetização das curvas smile dos ativos numa curva smile da carteira. 


\section{Apêndice A}

\section{Apêndice}

\section{A.1 Método de Laplace}

"Heuristic reasoning, rather than mathematical rigor, is often used to justify a procedure, or some extension of it. This [...] is mainly intended for mathematicians and scientists whose primary aim is to get answers to practical problems. [...] The philosophy $[. .$.$] is that, when solutions to non-standard problems$ are required, no procedure, be it rigorous or heuristic, should be scorned - a sentiment, succinctly summed up by Montaigne: 'In truth, and I am not afraid to admit it. I would, in need, light a candle to Saint Michael and another to his dragon' ". [Mur84]

A "Teoría de Análise Assintótica" está interessada em métodos que permitam obter aproximações analíticas ( séries de potências) de funções definidas por integrais. Os exemplos mais comuns de tais funções são dados pelas soluções de equações diferenciais e pelas funções de densidade.

Especificamente, analisamos uma generalização para $\mathbb{R}^{n}$ do método de Laplace. Este método recebe esse nome pois é aplicável em funções da forma

$$
f(x)=\int_{0}^{\infty} e^{-t x} \phi(x) d x \quad t \rightarrow \infty
$$


e, como é bem conhecido, se $x$ for um número complexo, $f(x)$ é a transformada de Laplace de $\phi$.

É importante para poder aplicar o método com sucesso entender a idéia subjacente. Para isto considerarnos o caso $\varkappa \in \mathbb{R}$, para poder ter uma visualização geométrica mais simples. Geralmente as funções estudadas são da forma:

$$
\begin{aligned}
& f(x)=\int_{0}^{T} e^{-t x} x^{\lambda} \phi(x) d x, \quad t \rightarrow \infty \text { e } \lambda>-1 ; \\
& g(x)=\int_{\alpha}^{\beta} e^{-t x^{2} \phi} \phi(x) d x, \quad t \rightarrow \infty .
\end{aligned}
$$

Nas expressões destas integrais resulta fácil ver que as maiores contribuições provem dos valores próximos ao $x=0$; istos são os valores que maximizam $-t x$ e $-t x^{2}$, portanto, maximizadores de $e^{-t x}$ e $e^{-t x^{2}}$. O incremento das áreas de integração é exemplificado na seguinte figura:

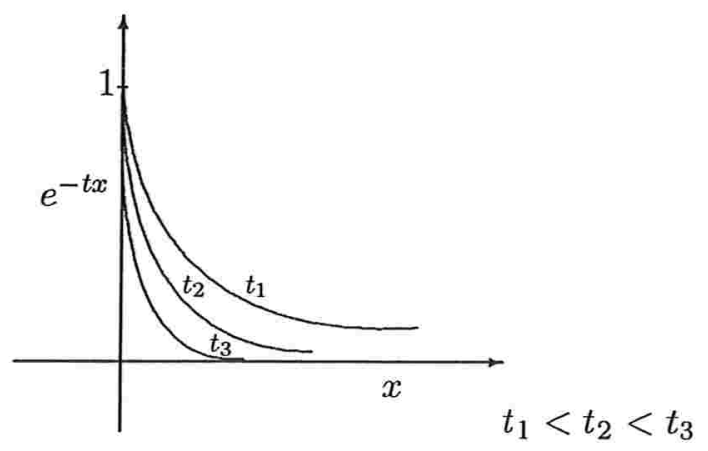

No desenho anterior podemos observar que quando $t$ aumenta, a base da área de integração diminui. Assim, a idéia que estamos querendo salientar é que a região próxima do $x=0$ é maior quanto maior é o $t$.

Tal idéia pode ser estendida para integrais da forma

$$
f(x)=\int_{\alpha}^{\beta} \phi(x) e^{t h(x)} d x .
$$

Um esquema da função anterior para $t \rightarrow \infty$ é o seguinte: 


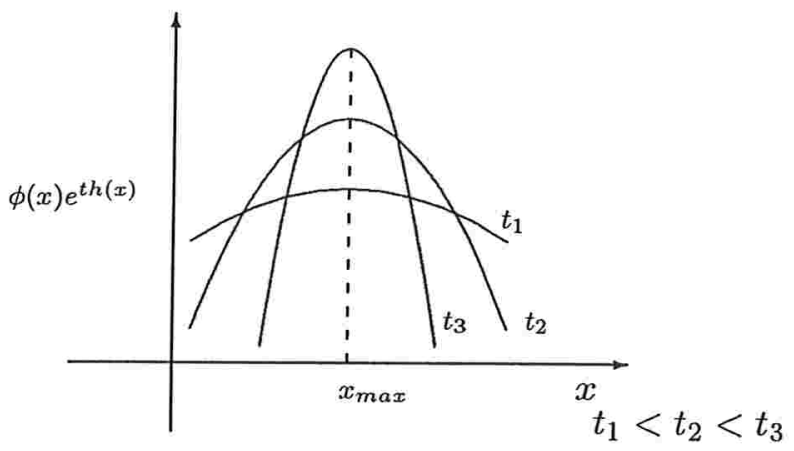

Neste caso observamos que as contribuições mais importantes estão dadas pelo ponto máximo de $h(x)$.

Agora estamos em condições de apresentar as definições e resultados teóricos que nós empregamos.

Definição A.1.1 Sejam $f$ e $g$ funções reais de variável real e $t_{0}$ um número real. Diremos que

1. $f(t)=O(g(t))$ quando $t \rightarrow t_{0}$ se existirem números reais $\epsilon>0$ e $\delta>0$ tais que

$$
\left|\frac{f(t)}{g(t)}\right| \leq \epsilon, \quad \forall t: 0<\left|t-t_{0}\right|<\delta ;
$$

2. $f(t)=O(g(t))$ quando $t \rightarrow \infty$ se existirem números reais $\epsilon>0 e$ $M>0$ tais que

$$
\left|\frac{f(t)}{g(t)}\right| \leq \epsilon, \quad \forall t: t>M .
$$

3. $f(t)=o(g(t))$ quando $t \rightarrow t_{0}$ se

$$
\lim _{t \rightarrow t_{0}}\left|\frac{f(t)}{g(t)}\right|=0
$$

Definição A.1.2 Uma seqüência finita ou infinita de funções $\left\{\phi_{n}(x)\right\}$, $n=1,2, \ldots$ é uma seqüência assintótica quando $x \rightarrow x_{0}$ se, para todo $n$

$$
\lim _{x \rightarrow x_{0}} \frac{\phi_{n+1}}{\phi_{n}}=0
$$


Subentende-se que $\phi_{n}$ não tem zeros em uma vizinhança de $x_{0}$ (em alguns casos apenas $x_{0}$ é zero).

Definição A.1.3 Se $\left\{\phi_{n}(x)\right\}$ é uma seqüência assintótica de funçôes quando $x \rightarrow x_{0}$, diremos que $\sum_{n=1} a_{n} \phi_{n}(x)$, onde $a_{n}$ são constantes, é uma expansão assintótica ou aproximação assintótica da função $f(x)$ se para cada $N$

$$
f(x)=\sum_{n=1}^{N} a_{n} \phi_{n}(x)+O\left(\phi_{N+1}(x)\right), \quad x \rightarrow x_{0} .
$$

Observamos que tais somas parciais usualmente não são convergentes, embora, $\left|f(x)-\sum_{n=1}^{N} a_{n} \phi_{n}(x)\right| \rightarrow 0$ para um $N$ fixo e $x \rightarrow x_{0}$. Mas não é o objetivo deste trabalho aprofundar nesse tema.

Teorema A.1.4 (Generalização do Lema de Watson para $\mathbb{R}^{\mathbf{n}}$ ) Seja $B_{\alpha} \subset \mathbb{R}^{n}$ uma região tal que $0 \in B_{\alpha}$ e $q_{\alpha}(\cdot)$ uma função na região. Assumimos que existem as seguintes constantes positivas:

(H1). $\epsilon_{1}, \delta_{1}$ e $M_{1}$ tais que se $\left\{\mathrm{x}:|\mathrm{x}|<\delta_{1}\right\} \subset B_{\alpha}$, então

$$
\int_{B_{\alpha}} e^{-M_{1}|\mathbf{x}|^{2}}\left|q_{\alpha}(\mathbf{x})\right| d \mathbf{x} \leq \epsilon_{1}
$$

(H2). $M_{2}$ tal que $\left|q_{\alpha}(0)\right| \leq M_{2}$ e para $1 \leq i \leq n\left|\frac{\partial q_{\alpha}(\mathrm{x})}{\partial_{x_{i}}}\right|_{\mathrm{x}=0} \mid \leq M_{2}$

(H3). $\epsilon_{2}$ e $\delta_{2}$ tais que para $|\mathrm{x}|<\delta_{2}$ temos

$$
\left|q_{\alpha}(\mathrm{x})-\left(q_{\alpha}(0)+\nabla q_{\alpha}(\mathbf{0}) \cdot \mathrm{x}\right)\right| \leq \epsilon_{2}
$$

Logo, para $\alpha \rightarrow \infty$,

$$
\left(\frac{\alpha}{2 \pi}\right)^{n / 2} \int_{B_{\alpha}} e^{-\frac{\alpha}{2}|\mathbf{x}|^{2}} q_{\alpha}(\mathrm{x}) d \mathrm{x}=q_{\alpha}(0)+O\left(\alpha^{-1}\right) .
$$




\section{Demonstração.}

Seja $\delta=\min \left\{\delta_{1}, \delta_{2}\right\}$,

$$
\begin{aligned}
& \left(\frac{\alpha}{2 \pi}\right)^{n / 2} \int_{B_{\alpha}} e^{-\frac{\alpha}{2}|\mathbf{x}|^{2}} q_{\alpha}(\mathbf{x}) d \mathbf{x}= \\
& =\underbrace{\left(\frac{\alpha}{2 \pi}\right)^{n / 2} \int_{B_{\delta}} e^{-\frac{\alpha}{2}|\mathbf{x}|^{2}} q_{\alpha}(\mathrm{x}) d \mathbf{x}}_{B}+\underbrace{\left(\frac{\alpha}{2 \pi}\right)^{n / 2} \int_{\mathbb{R}^{n} \backslash B_{\delta}} e^{-\frac{\alpha}{2}|\mathbf{x}|^{2}} q_{\alpha}(\mathrm{x}) d \mathbf{x}}_{A}
\end{aligned}
$$

Estudemos primeiro o integrando $A$ utilizando a hipótese (H1)

$$
\begin{aligned}
& \left|\left(\frac{\alpha}{2 \pi}\right)^{n / 2} \int_{\mathbb{R}^{n} \backslash B_{\delta}} e^{-\left(\frac{\alpha}{2}-M_{1}+M_{1}\right)|\mathbf{x}|^{2}} q_{\alpha}(\mathrm{x}) d \mathrm{x}\right| \\
& \leq\left(\frac{\alpha}{2 \pi}\right)^{n / 2} e^{-\left(\frac{\alpha}{2}-M_{1}\right) \delta^{2}} \int_{\mathbb{R}^{n} \backslash B_{\delta}} e^{-M_{1}|\mathbf{x}|^{2}}\left|q_{\alpha}(\mathrm{x})\right| d \mathrm{x} \\
& \leq\left(\frac{\alpha}{2 \pi}\right)^{n / 2} e^{-\left(\frac{\alpha}{2}-M_{1}\right) \delta^{2}} \epsilon_{1} \\
& =O\left(\alpha^{-1}\right) .
\end{aligned}
$$

Para o integrando $B$, pela hipótese (H3) podemos afirmar que

$$
B=\left(\frac{\alpha}{2 \pi}\right)^{n / 2} \int_{B_{\delta}} e^{-\frac{\alpha}{2}|\mathbf{x}|^{2}}\left(q_{\alpha}(0)+\nabla q_{\alpha}(0) \cdot \mathrm{x}\right) d \mathbf{x}+O\left(\alpha^{-1}\right) .
$$

Por outro lado

$$
\begin{aligned}
& \left(\frac{\alpha}{2 \pi}\right)^{n / 2} \int_{B_{\delta}} e^{-\frac{\alpha}{2}|\mathbf{x}|^{2}}\left(q_{\alpha}(0)+\nabla q_{\alpha}(0) \cdot \mathbf{x}\right) d \mathbf{x}= \\
& =q_{\alpha}(0)\left(\frac{\alpha}{2 \pi}\right)^{n / 2} \int_{\mathbb{R}^{n}} e^{-\frac{\alpha}{2}|\mathbf{x}|^{2}} d \mathbf{x}+\left.\sum_{i=1}^{n} \frac{\partial q_{\alpha}}{\partial x_{i}}\right|_{\mathbf{x}=0}\left(\frac{\alpha}{2 \pi}\right)^{n / 2} \int_{\mathbb{R}^{n}} e^{-\frac{\alpha}{2}|\mathbf{x}|^{2}} x_{i} d \mathbf{x} \\
& -q_{\alpha}(0)\left(\frac{\alpha}{2 \pi}\right)^{n / 2} \int_{\mathbb{R}^{n} \backslash B_{\delta}} e^{-\frac{\alpha}{2}|\mathbf{x}|^{2}} d \mathbf{x}-\left.\sum_{i=1}^{n} \frac{\partial q_{\alpha}}{\partial x_{i}}\right|_{\mathbf{x}=0}\left(\frac{\alpha}{2 \pi}\right)^{n / 2} \int_{\mathbb{R}^{n} \backslash B_{\delta}} e^{-\frac{\alpha}{2}|\mathbf{x}|^{2}} x_{i} d \mathbf{x} \\
& =q_{\alpha}(0)\left(\frac{1}{2 \pi}\right)^{n / 2} \int_{\mathbb{R}^{n}} e^{-\frac{1}{2}|\mathbf{y}|^{2}} d \mathbf{y}+\left.\sum_{i=1}^{n} \frac{\partial q_{\alpha}}{\partial x_{i}}\right|_{\mathbf{x}=0}\left(\frac{1}{2 \pi}\right)^{n / 2} \int_{\mathbb{R}^{n}} e^{-\frac{1}{2}|\mathbf{y}|^{2}} y_{i} d \mathbf{y} \\
& +O\left(\alpha^{-1}\right) .
\end{aligned}
$$

Tendo utilizado a mudança de variáveis $y=\sqrt{\alpha} x$ e a hipótese (H2). 
Dado que

$$
\int_{\mathbb{R}^{n}} e^{-\frac{|\mathbf{y}|^{2}}{2}} y_{i} d \mathbf{y}=0
$$

(ver lema A.1.5), concluímos a prova do teorema.

Lema A.1.5

$$
\int_{\mathbb{R}^{n}} \exp \left(-\frac{|\mathrm{x}|^{2}}{2}\right) x_{i} d \mathrm{x}=0, \quad i=1, \ldots, n .
$$

\section{Demonstração.}

Para o caso unidimensional o lema é trivial, pois o integrando é antisimétrico.

Para o caso $n$-dimensional, usamos Fubini para reduzir ao caso unidimensional:

$$
\begin{aligned}
\int_{\mathbb{R}^{n}} e^{-\frac{|\mathrm{x}|^{2}}{2}} x_{i} d \mathrm{x} & =\int_{-\infty}^{\infty} e^{-\frac{x_{1}^{2}}{2}} d x_{1} \cdots \int_{-\infty}^{\infty} e^{-\frac{x_{i}^{2}}{2}} x_{i} d x_{i} \cdots \int_{-\infty}^{\infty} e^{-\frac{x_{n}^{2}}{2}} d x_{n} \\
& =0
\end{aligned}
$$

pois o produto anterior possui um integrando nulo.

A generalização do Lema de Watson pode ser estendida para derivadas de ordens $k>1$ obtendo:

$$
\begin{aligned}
& \left(\frac{\alpha}{2 \pi}\right)^{n / 2} \int_{B_{\alpha}} e^{-\frac{\alpha}{2}|x|^{2}} q_{\alpha}(x) d x= \\
& =q_{\alpha}(0)+\left.\frac{1}{\alpha} \sum_{i_{1}, i_{2}=1}^{n} \frac{\partial^{2} q_{\alpha}(x)}{\partial_{x_{i_{1}}} \partial_{x_{i_{2}}}}\right|_{x=0} E_{\left(i_{1}, i_{2}\right)}+\cdots \\
& +\left.\frac{1}{\alpha^{K}} \sum_{i_{1}, \ldots, i_{2 K}=1}^{n} \frac{\partial^{2 K} q_{\alpha}(x)}{\partial_{x_{i_{1}}} \cdots \partial_{x_{i_{2 K}}}}\right|_{x=0} E_{\left(i_{1}, \ldots, i_{2 K}\right)} \\
& +O\left(\alpha^{-\frac{k+1}{2}}\right)
\end{aligned}
$$

na qual

$$
E_{\left(i_{1}, \ldots, i_{j}\right)}=(2 \pi)^{-n / 2} \int_{\mathbb{R}^{n}} \exp \left(\frac{-|x|^{2}}{2}\right) x_{i_{1}} \cdots x_{i_{j}} d x
$$

e $K \equiv\lfloor k / 2\rfloor$. 


\section{A.2 Algoritmos}

Esquema dos passos para os cálculos numéricos ( determinação de $\left.\sigma_{I}(B)\right)$

[passo 1 ] Para $i, j=1, \ldots, n$, determinar os coeficientes de correlação $\rho_{i j}$ entre as ações $i$ e $j$ utilizando dados históricos.

[passo 2 ] Determinar (simultaneamente) as curvas das volatilidades implícitas de cada ação.

[passo 3 ] Calcular os seguintes valores:

$$
\begin{aligned}
\bar{x} & =\ln \left(\frac{B}{\sum_{i=1}^{n} w_{i} S_{i}(0)}\right) \\
p_{i}(0,0) & =\frac{w_{i} S_{i}(0)}{\sum_{i=1}^{n} w_{i} S_{i}(0)} \quad i=1, \ldots, n .
\end{aligned}
$$

[passo 4 ] Determinar

$$
\sigma_{0}^{2}(\mathbf{0})=\sum_{i, j=1}^{n} \rho_{i j} \sigma_{i}^{I}\left(S_{i}(0)\right) \sigma_{j}^{I}\left(S_{j}(0)\right) p_{i}(0,0) p_{j}(0,0) .
$$

[passo 5 ] Para $i=1, \ldots, n$ calcular

$$
\begin{aligned}
x_{i}^{*} & =\frac{\bar{x}}{\sigma_{0}^{2}(0)} \sum_{j=1}^{n} \rho_{i j} p_{j}(0,0) \sigma_{j}^{I}\left(S_{j}(0)\right) \\
S_{i}^{*} & =S_{i}(0) e^{x_{i}^{*}} \\
p_{i}\left(0, \mathrm{x}^{*}\right) & =\frac{w_{i} S_{i}^{*}}{\sum_{j=1}^{n} w_{j} S_{j}^{*}} .
\end{aligned}
$$

[passo 6 ] Aproximar as volatilidades locais de cada ativo, isto é, calcular os seguintes valores

$$
\alpha_{i} \equiv 2 \sigma_{i}^{I}\left(S_{i}^{*}\right)-\sigma_{i}^{I}\left(S_{i}(0)\right) \quad i=1, \ldots, n .
$$

[passo 7 ] Obter a volatilidade implícita do índice $B$, dada por

$$
\sigma_{I}(B)=\frac{\sqrt{\sum_{i, j=1}^{n} \rho_{i j} p_{i}\left(0, \mathrm{x}^{*}\right) p_{j}\left(0, \mathrm{x}^{*}\right) \alpha_{i} \alpha_{j}}+\sigma_{0}(0)}{2} .
$$




\section{Referências Bibliográficas}

[Ave02] M. Avellaneda, D. Boyer-Olson, J. Busca, e P. Friz.

Reconstruction of volatility: Pricing index options using the steepest-descent approximation. (Outubro de 2002).

fonte:www.math.nyu.edu/faculty/avellane.

[BBF02] H. Berestycki, J. Busca, e I. Florent. Asymptotics and calibration of local volatility models. Quantitative Finance, 2(1):6169, 2002.

[Cop65] E. T. Copson. Asymptotic Expansions. Cambridge University, Press., 1965.

[Gat01] J. Gatheral. Stochastic volatility and local volatility: Case studies in financial modeling. Notas de aula, M.S. Program in Math. Finance, NYU, 2001.

[Jen95] J. Ledet Jensen. Saddlepoint Approximation. Oxford Science Publications, 1995.

[Lue84] David G. Luenberger. Linear and Nonlinear Programming. Addison-Wesley, 2 edição, 1984.

[Mur84] J. D. Murray. Asymptotic Analysis. Applied Mathematical Sciencies 48. Springer-Verlag, 1984. 
[Øks00] Bernt $\varnothing \mathrm{ksendal}$. Stochastic Differential Equations: An Introduction with Applications. Springer-Verlag, $5^{\circ}$ edição, 2000.

[Var67a] S. R. S. Varadhan. Diffusion processes in a small time interval. Commun. Pure Appl. Math., 20, 1967.

[Var67b] S. R. S. Varadhan. On the behaviour of the fundamental solution of the heat equation with variable coefficients. Commun. Pure Appl. Math., 20, 1967. 